\title{
Long non-coding RNA Neat1 regulates adaptive behavioural response to stress in mice
}

\author{
Michail S. Kukharsky $\mathbb{1}^{1,2}$, Natalia N. Ninkina ${ }^{1,2}$, Haiyan An ${ }^{1,3}$, Vsevolod Telezhkin ${ }^{4}$, Wenbin Wei, ${ }^{5,6}$, \\ Camille Rabesahala de Meritens, ${ }^{3}$, Johnathan Cooper-Knock ${ }^{5}$, Shinichi Nakagawa', Tetsuro Hirose ${ }^{6}$, \\ Vladimir L. Buchman ${ }^{1,2}$ and Tatyana A. Shelkovnikova (1) $\mathbb{1}^{1,2,3}$
}

\begin{abstract}
NEAT1 is a highly and ubiquitously expressed long non-coding RNA (IncRNA) which serves as an important regulator of cellular stress response. However, the physiological role of NEAT1 in the central nervous system (CNS) is still poorly understood. In the current study, we addressed this by characterising the CNS function of the Neat1 knockout mouse model (Neat $1^{-1-}$ mice), using a combination of behavioural phenotyping, electrophysiology and expression analysis. RNAscope ${ }^{\circledR}$ in situ hybridisation revealed that in wild-type mice, Neat1 is expressed across the CNS regions, with high expression in glial cells and low expression in neurons. Loss of Neat1 in mice results in an inadequate reaction to physiological stress manifested as hyperlocomotion and panic escape response. In addition, Neat ${ }^{-1-}$ mice display deficits in social interaction and rhythmic patterns of activity but retain normal motor function and memory. Neat ${ }^{-1}$ mice do not present with neuronal loss, overt neuroinflammation or gross synaptic dysfunction in the brain. However, cultured Neat $1^{-1}$ neurons are characterised by hyperexcitability and dysregulated calcium homoeostasis, and stressinduced neuronal activity is also augmented in Neat $1^{-1-}$ mice in vivo. Gene expression analysis showed that Neat1 may act as a weak positive regulator of multiple genes in the brain. Furthermore, loss of Neat1 affects alternative splicing of genes important for the CNS function and implicated in neurological diseases. Overall, our data suggest that Neat1 is involved in stress signalling in the brain and fine-tunes the CNS functions to enable adaptive behaviour in response to physiological stress.
\end{abstract}

\section{Introduction}

Long noncoding RNAs (lncRNAs) are an arbitrary group comprising non-protein coding transcripts longer than 200 nucleotides. The majority of lncRNAs display tissue-specific, cell-specific, and context-specific expression, and only few of them are ubiquitous and highly expressed. The prominent member of the latter group is Nuclear Paraspeckle Assembly Transcript 1 (NEAT1), a nuclear-retained lncRNA with important roles in cellular (patho)physiology and one of the most intensely studied lncRNAs. NEAT1 contributes to various cellular

\footnotetext{
Correspondence: Tatyana A. Shelkovnikova (shelkovnikovat@cardiff.ac.uk) ${ }^{1}$ School of Biosciences, Cardiff University, Cardiff CF10 3AX, UK

${ }^{2}$ Institute of Physiologically Active Compounds of Russian Academy of Sciences, Chernogolovka 142432, Russian Federation

Full list of author information is available at the end of the article
}

processes, from regulation of transcription and chromatin active state to miRNA biogenesis (reviewed in the ref. ${ }^{1}$ ). NEAT1 locus produces two transcripts, NEAT1_1 and NEAT1_2. The longer NEAT1 isoform, NEAT1_2, is essential for the assembly of nuclear bodies termed paraspeckles $^{2,3}$, whereas NEAT1_1, albeit also a paraspeckle component, is dispensable for their formation and likely plays various paraspeckle-independent roles ${ }^{4}$. NEAT1 expression is elevated in stressed cells ${ }^{5}$, such as those subjected to hypoxia ${ }^{6}$, viral infection ${ }^{7}$, heat shock ${ }^{8}$, mitochondrial stress ${ }^{9}$ or proteasome inhibition ${ }^{10}$. NEAT1 transcripts have been shown to regulate epigenetic marks on histones ${ }^{11,12}$. Changes in NEAT1 levels is a recurrent theme in neoplasias, and the gene is a hotspot for mutations in several types of cancer ${ }^{1}$.

\section{(c) The Author(s) 2020}

(c) (i) Open Access This article is licensed under a Creative Commons Attribution 4.0 International License, which permits use, sharing, adaptation, distribution and reproduction cc) in any medium or format, as long as you give appropriate credit to the original author(s) and the source, provide a link to the Creative Commons license, and indicate if changes were made. The images or other third party material in this article are included in the article's Creative Commons license, unless indicated otherwise in a credit line to the material. If material is not included in the article's Creative Commons license and your intended use is not permitted by statutory regulation or exceeds the permitted use, you will need to obtain permission directly from the copyright holder. To view a copy of this license, visit http://creativecommons.org/licenses/by/4.0/. 
According to the genotype-tissue expression (GTEx) database, NEAT1 is expressed ubiquitously in the human body, including in the CNS (Supplementary Fig. S1). Despite NEAT1 expression in the CNS is lower than other organs and tissues, NEAT1 transcripts are induced under specific conditions, e.g., by augmented neuronal activity ${ }^{13}$, pointing to important regulatory roles of this lncRNA in the brain. Because levels of NEAT1_2 in the intact brain are almost negligible ${ }^{14}$, NEAT1_1 can be considered as the main functional NEAT1 transcript in the CNS tissue under basal conditions. NEAT1 is able to modulate neuronal excitability, where its acute downregulation renders neurons more excitable ${ }^{13}$. Altered NEAT1 expression has been reported in all major neurodegenerative and psychiatric diseases, including frontotemporal dementia (FTD), Alzheimer's, Huntington's and Parkinson's diseases, amyotrophic lateral sclerosis (ALS), epilepsy, traumatic brain injury and schizophrenia (reviewed in the ref. ${ }^{15}$ ). However, mechanisms of NEAT1 transcripts involvement in the neurological conditions are still poorly understood, primarily because our knowledge of their function(s) is the CNS is still scarce. We still lack a clear picture of what and how NEAT1 contributes to neuronal function, especially at the organismal level.

Neat1 knockout mouse strain was generated in 2011 through disruption of the Neat1 promoter sequences common for both Neat1 isoforms, and these mice appeared to be viable and superficially normal ${ }^{14}$. However, subsequent more detailed studies revealed hormone dysfunction and decreased fertility of Neat1 knockout females ${ }^{16}$, confirming an important role for Neat1 transcripts in specific physiological processes. Neat1 knockout mice do not present with an overt neurological phenotype, however, neuronal deficits in these mice may only manifest under certain conditions such as the physiological stress experienced during pregnancy.

In the current study, we interrogated Neat1 function in the mammalian CNS using this mouse line. We show that loss of Neat1 perturbs normal behavioural responses of mice specifically under conditions of stress. This phenotype is not due to neuronal loss, neuroinflammation or gross changes in synaptic functions but rather can be attributed to altered neuronal excitability and changes in the alternative splicing of genes important for the CNS function. Our data suggest that Neat1 fine-tunes the CNS function under stressful conditions at the organismal level, which is consistent with the current view of NEAT1 as stress-responsive transcripts at the cellular level.

\section{Materials and methods}

Mouse colonies and genotyping

Generation of the Neat1 ${ }^{-1-}$ mouse strain has been described previously ${ }^{14}$. The strain was maintained on the C57Bl/6J genetic background by backcrossing with wild-type mice from Charles River, UK. Primers used for mouse genotyping by PCR were $5^{\prime}$-CTAGTGGTGGGGAGGCAGT- $3^{\prime}$ and $5^{\prime}$-AGCAGGGATAGCCTGGT CTT-3 ${ }^{\prime}$. Homozygous (Neat $1^{-/-}$and wild-type $\left(\right.$Neat $1^{+/+}$) mice were obtained by intercrossing heterozygous $\left(\right.$ Neat $\left.^{+/-}\right)$mice. Experimental animals were housed individually, at $12 \mathrm{~h}$ light $/ 12 \mathrm{~h}$ dark cycle, with food and water supplied ad libitum. Before behavioural testing, the animals were placed in the testing room in their home cages for $1 \mathrm{~h}$ for habituation. All work on animals was carried out in accordance with the United Kingdom Animals (Scientific Procedures) Act (1986).

\section{Behavioural testing \\ Locomotor activity}

Spontaneous locomotor activity was assessed in the home-like cage $(\mathrm{HC})$ equipped with infra-red beams to monitor horizontal movements over a period of $36 \mathrm{~h}$, under the $12 \mathrm{~h}$ light $/ 12 \mathrm{~h}$ dark cycle. Number of beam breaks was recorded at 15-min intervals. The same level of illumination (as in the room used for normal housing (15-20 lux) was maintained during the day phase of the test. Locomotor activity under bright light conditions (600 lux) was recorded for $1 \mathrm{~h}$ using Ugo Basile 47420 multiple activity cage.

\section{Elevated plus maze test}

The test was performed on a standard plus-shaped apparatus under bright light (400 lux). Each mouse was placed in the central square of the maze facing one of the open arms and was allowed to freely explore the maze for $5 \mathrm{~min}$. Video recordings were analysed to determine the duration and frequency of entries into open and closed arms, number of head dips and stretch-attend postures $^{17,18}$.

\section{$Y$-maze test}

Working memory was assessed using a Y-maze apparatus with arms measuring $40 \times 6 \times 20 \mathrm{~cm}(\mathrm{~L} \times \mathrm{W} \times \mathrm{H})$. The test was conducted under dim light conditions (15-20 lux). Forced alternations were assessed in the twotrial test ${ }^{19,20}$. Briefly, mice were initially allowed to explore two arms of maze for 5 min with the third arm blocked. After a $30 \mathrm{~min}$ inter-trial interval, the second trial was conducted, where mice were allowed to explore all three arms for $5 \mathrm{~min}$. An arm entry was recorded when more than half of the mouse body crossed the border between two arms. Number of entries and time spent in each arm were registered.

\section{Resident-intruder test}

To assess aggression and social interaction, the standard protocol of resident-intruder test was used ${ }^{21,22}$. Animals were maintained without bedding changed for 1 week 
before the task. The cage top was removed and the male intruder was placed in the cage for $5 \mathrm{~min}$. Older and heavier $\mathrm{C} 57 \mathrm{Bl} / 6 \mathrm{~J}$ males were used as intruders (single time for each animal). The number of attacks and total time spent in a physical interaction were recorded.

\section{Social odour test}

Social odours were collected from a home cage where five C57Bl/ 6 adult male mice had been housed for 6 days without bedding change. The bottom of the cage was wiped with a cotton swab in a zig-zag pattern to collect social odour. Mice were left to habituate in a new cage in the presence of an unused cotton swab for 30 min prior to test. Subsequently, mice were exposed to a new cotton swab without odour, followed by a cotton swab with a social odour. The amount of time spent sniffing each swab over a period of $2 \mathrm{~min}$ was recorded.

\section{Restraint stress}

To induce $c F o s$ expression, mice were exposed to a 60 min restraint stress. Animals were closed into custommade cylindrical polycarbonate tubes with ventilation holes measuring $12.7 \mathrm{~cm}$ long and $3.8 \mathrm{~cm}$ in diameter, which allowed to significantly restrict their movements. Control animals were left undisturbed in their home cages in the same procedure room. After the restraint, mice were returned to their home cages for $60 \mathrm{~min}$. Control and experimental mice were euthanised immediately after, and brain tissue was fixed for analysis as described below.

\section{RNA isolation and gene expression analysis in mouse tissue}

Whole cortices were carefully dissected from freshly removed brains on ice and thoroughly homogenised to ensure equal representation of cells from different cortex regions in the experimental samples. Total RNA was extracted using PureLink total RNA extraction kit (Life Technologies) and possible DNA contamination was removed using RNase free DNase kit (Qiagen). cDNA synthesis was performed on $500 \mathrm{ng}$ of total RNA using SuperScript IV reverse transcriptase (Life Technologies) and random hexamer primers (Promega). Quantitative real-time PCR was run in triplicate on an StepOne ${ }^{\mathrm{TM}}$ realtime PCR instrument and data were analyzed using StepOne $^{\mathrm{Tm}}$ Software v2.0 (all Applied Biosystems, Life Technologies). Gapdh was used for normalisation. Primer seqeunces were as follows: Neat1 total 5'-TGGAGA TTGAAGGCGCAAGT $3^{\prime}$ and $5^{\prime}$-ACCACAGAAGAGG AAGCACG-3'; Neat1_2 5'-AACTACCAGCAATTCC GCCA-3' and 5'-GAGCTCGCCAGGTTTACAGT-3'; Malat1 5'-GAGCTCGCCAGGTTTACAGT-3' and 5'-AA CTACCAGCAATTCCGCCA-3'; Gapdh 5'-AAGAGGG ATGCTGCCCTTAC-3' and 5'-TACGGCCAAATCCG
TTCACA-3'; Gabrr2 5'-ACATCCAAGCCAAGCCAT TTG-3' and 5'-CATGGTGAAGTCGTGGTCCT-3'; Gabra1 5' $5^{\prime}$-TATGGACAGCCCTCCCAAGA-3' and $5^{\prime}-\mathrm{T}$ ACACGCTCTCCCAAACCTG-3'; Gabra2 5'-ACAGATT CAAAGCCACTGGAGG-3 ${ }^{\prime}$ and 5'-TCTTCTTGTT GCCAAAGCTG-3'; Rfk 5'-GGCCACATTGAGAATTTC CCC- $3^{\prime}$ and $5^{\prime}$-GGTGGTGAGCATTGGATGGA-3'.

\section{RNA sequencing and analysis}

Total RNA was prepared as described above and its quality examined using Agilent 4200 TapeStation. RNA sequencing was performed at the School of Biosciences Genomics Research Hub. Libraries were prepared using the TruSeq stranded total kit (Illumina). For the main experiment (in 2-month old mice), paired-end sequencing was carried out on Illumina NextSeq500 (read length: $75 \mathrm{bp}$; coverage $\sim 50$ million reads/sample). For the additional experiment (low-coverage, in 1-month-old mice) single-end sequencing was performed instead (read length: 75 bp; coverage $\sim 25$ million reads/sample). Reads were aligned to the mouse reference genome (GRCm38) using STAR ${ }^{23}$, and Fragments Per Kilobase of transcript per Million mapped reads (FPKM) values were obtained using DESeq2 24 . Sequencing reads were viewed in the IGV browser ${ }^{25}$. Ensembl IDs for genes identified by DESeq2 were mapped to known genes and tested for GO term enrichment using DAVID 6.8 online tool (https://david.ncifcrf.gov/). Differential splicing events were identified using the Leafcutter pipeline ${ }^{26}$. GO term and pathway enrichment analysis of differentially spliced genes was performed using the Enrichr online tool (https://amp.pharm.mssm.edu/Enrichr/) 27,28 .

\section{Western blotting}

SDS-PAGE loading buffer was added to homogenised samples, followed by denaturation at $100{ }^{\circ} \mathrm{C}$ for $5 \mathrm{~min}$. After SDS-PAGE using Mini-Protean ${ }^{\circledR}$ precast gels (Bio$\mathrm{Rad})$, proteins were transferred to PVDF or nitrocellulose membrane (GE Healthcare) by semi-dry blotting. Membranes were blocked in $4 \%$ milk in TBST, incubated in primary antibody at $4{ }^{\circ} \mathrm{C}$ overnight and in HRPconjugated secondary antibody (GE Healthcare) for $1.5 \mathrm{~h}$ at RT. For detection, WesternBright Sirius kit (Advansta) and Bio-Rad ChemiDoc ${ }^{\mathrm{TM}}$ Gel Imaging System were used. Equal loading was confirmed by re-probing membranes with an antibody against beta-actin. Protein levels were quantified using the band analysis tool of Image J software (https://imagej.nih.gov/ij/).

\section{RNA in situ hybridisation and immunohistochemistry in mouse tissue}

Whole mouse brains and spinal cord sections were fixed in $4 \%$ PFA overnight and embedded in paraffin wax. Eight $\mu \mathrm{m}$ thick sections were mounted on poly-L-lysine coated 
slides (ThermoScientific). For RNAscope ${ }^{\circledR}$ ISH analysis, Neat1_1 (440351), with the target region 1416-2381 in mouse Neat1, and Neat1_2 (538761), with the target region 7378-8453, probes (Advanced Cell Diagnostics) were used according to manufacturer's instructions. For Nissl staining, sections were incubated in $0.5 \%$ Cresyl Violet Acetate solution (C5042, Sigma) and differentiated in acidified ethanol. Immunohistochemistry with 3,3'diaminobenzidine (DAB) as a substrate was performed as described earlier ${ }^{29}$. Images were taken using Leica DMRB microscope or Olympus BX40 slide scanner $(\times 20$ magnification). The images of sagittal sections for the same brain regions $(1000 \pm 200 \mu \mathrm{m}$ from the midline) for all animals were obtained. Quantification of neuron numbers was performed using the 3D Object Counter plugin of ImageJ using an equally sized region of interest (ROI) for the cortex zone incorporating all cortex layers.

\section{Primary antibodies}

Primary antibodies used for western blot and immunohistochemistry: GFAP (rabbit polyclonal, G9269, Sigma); Iba1 (rabbit monoclonal, ab178846, Abcam); PSD95 (DLG4) (rabbit polyclonal, 20665-1-AP, Proteintech), synaptophysin (mouse monoclonal, 611880, BD Biosciences); riboflavin kinase (rabbit polyclonal, 15813-1-AP, Proteintech); GAD67 (rabbit polyclonal, 10408-1-AP, Proteintech); cFos (mouse monoclonal, 66590-1-Ig, Proteintech); beta-actin (mouse monoclonal A5441, Sigma).

\section{Analysis of NEAT1_2 induction in stressed human ES cell derived neurons}

Motor neuron enriched cultures were obtained by chemically induced differentiation of H9 human embryonic stem ( $\mathrm{hES}$ ) cells, as described previously ${ }^{30}$. Briefly, hES cells were maintained in mTESR-Plus media (Stemcell Technologies) on plates coated with Matrigel $^{\circledast}$ (Corning). Cultures were tested for mycoplasma contamination using MycoAlertTM Mycoplasma Detection Kit, Lonza, cat \# LT07-218 and determined to be negative. Confluent cultures were subjected to neuronal patterning in advanced DMEM/F12 (ADF) medium supplemented with SB431542 $(10 \mu \mathrm{M}$, Abcam) from day 1; and purmorphamine $(1 \mu \mathrm{M}$, Cayman Chemicals) and retinoic acid $(0.1 \mu \mathrm{M}$, Sigma) from day 4 . On day 16 , neural progenitors were dissociated using Accutase $^{\circledast}$ and plated onto Matrigel $^{\circledast}$ coated dishes and cultured in ADF with GlutaMAX, penicillin-streptomycin, B27 (12587-010), N2 (all Life Technologies) and BDNF (Miltenyi, $10 \mathrm{ng} / \mathrm{ml}$ ). On day 23, neurons were re-plated using Accutase $^{\circledR}$ on coverslips at desired density and maintained in 50/50 mixture of ADF/Neurobasal A with the above supplements until Day 36. Neurons were treated with $0.5 \mathrm{mM}$ or $0.05 \mathrm{mM}$ NaAsO2 (Sigma), or $10 \mu \mathrm{M}$ MG132 (Sigma). Recovery took place in fresh culturing medium. NEAT1_2 induction was analysed by RNA-FISH using a commercial probe (Stellaris ${ }^{\circledR}$, Biosearch Technologies, human NEAT1 middle segment, SMF-2037-1) according to manufacturer's protocol. Neurite network was visualised using anti-neurofilament $\mathrm{H}$ immunostaining (rabbit polyclonal antibody, N4142, Sigma) as described elsewher ${ }^{30}$. Nuclei were visualised with DAPI (Sigma). Fluorescent images were taken using BX57 fluorescent microscope equipped with ORCA-Flash 4.0 camera (Hamamatsu) and cellSens Dimension software (Olympus). RNA isolation and qRTPCR analysis were performed as described above for mouse tissue, using the following human-specific primers: NEAT1 total: 5'- CTCACAGGCAGGGGAAATGT-3' and $5^{\prime}$-AACACCCACACCCCAAACAA-3'; NEAT1_2: $5^{\prime}$-TGTGTGTGTAAAAGAGAGAAGTTGTGG- $3^{\prime}$ and 5' - AGAGGCTCAGAGAGGACTGTAACCT -3'; GAPD $\mathrm{H}$ : 5'-TCGCCAGCCGAGCCA-3' and 5'-GAGTTAAA AGCAGCCCTGGTG -3'.

\section{Primary mouse hippocampal cultures}

Primary cultures of mouse hippocampal neurons were prepared from P1 litters of wild-type and Neat ${ }^{-/-}$mice as described previously ${ }^{31}$. Neurons were transfected with pEGFP-C1 plasmid using Lipofectamine2000 (Life Technologies) and imaged $24 \mathrm{~h}$ post-transfection, using BX61 microscope equipped with F-View II camera and CellF software. Day in vitro (DIV) 7 neurons were used for analysis. Neurite analysis was performed using "Sholl Analysis" and "Simple Neurite Tracer" plugins of ImageJ.

\section{Electrophysiological recordings and analysis}

Patch-clamp recordings of membrane potential and transmembrane current were performed using conventional whole-cell configuration on DIV7 neurons isolated from $\mathrm{Neat1}^{+/+}$and $\mathrm{Neat1}^{-/-}$mice and cultured in vitro ${ }^{32}$. Electrophysiological signals were recorded with Axopatch 200B amplifier interfaced to a computer running pClamp 9 using a Digidata 1322A A/D (Molecular Devices). All electrophysiological studies were performed at a controlled room temperature of $22 \pm 0.5^{\circ} \mathrm{C}$. Recordings were digitised at $10 \mathrm{kHz}$ and low-pass filtered at $2 \mathrm{~Hz}$ using an 8-pole Bessel filter. The standard bath solution (pH 7.4) contained: $135 \mathrm{mM} \mathrm{NaCl}, 5 \mathrm{mM} \mathrm{KCl}, 1.2 \mathrm{mM}$ $\mathrm{MgCl}_{2}, 1.25 \mathrm{mM} \mathrm{CaCl}_{2}, 10 \mathrm{mM}$ D-glucose, $5 \mathrm{mM}$ HEPES. The standard pipette solution ( $\mathrm{pH}$ 7.2) contained: $117 \mathrm{mM} \mathrm{KCl}, 10 \mathrm{mM} \mathrm{NaCl}, 11 \mathrm{mM}$ HEPES, $2 \mathrm{mM} \mathrm{Na} 2-$ ATP, $2 \mathrm{mM}$ Na-GTP, $1.2 \mathrm{mM}$ Na2-phosphocreatine, $2 \mathrm{mM} \mathrm{MgCl}_{2}, 1 \mathrm{mM} \mathrm{CaCl} 2$ and $11 \mathrm{mM}$ EGTA. Mean resting membrane potential $(\mathrm{Vm})$ of the neurons were determined during $120 \mathrm{~s}$ gap-free recording periods in current clamp mode $(I=0 \mathrm{pA})$. Spike analysis was performed on the first iAP using Clampfit 9. $\mathrm{Na}+$ currents were recorded using a standard voltage-step protocol, as described elsewhere ${ }^{33}$. 


\section{$\mathrm{Ca}^{2+}$ imaging}

Imaging was performed using Olympus IX71 inverted microscope and fluorescence source (Xenon arc or LED), alongside with a rapid perfusion system. Neurons were loaded with Fura-2 AM (ThermoFisher, $4 \mu \mathrm{M}$ final concentration) in full media for 45 min under standard culturing conditions. Cells were transferred into basal recording solution for imaging $(135 \mathrm{mM} \mathrm{NaCl}, 5 \mathrm{mM} \mathrm{KCl}$, $2 \mathrm{mM} \mathrm{CaCl}, 1 \mathrm{mM} \mathrm{MgCl}_{2}, 10 \mathrm{mM}$ glucose, $10 \mathrm{mM}$ HEPES). Prior to $\mathrm{KCl}$ stimulation, basal recordings were taken for $60 \mathrm{~s}$. $\mathrm{KCl}(60 \mathrm{mM})$ solution was injected at this point, and recording continued for further $300 \mathrm{~s} . \Delta F / F 0$ was calculated as $F(340 \mathrm{~nm}) / F(380 \mathrm{~nm})$ and used to prepare the plot.

\section{Experimental design}

To generate experimental cohorts, heterozygous mice $\left(\mathrm{Neat}^{+/-}\right)$on the $\mathrm{C} 57 \mathrm{Bl} / 6 \mathrm{~J}$ background were used. After genotyping the progeny, animals were randomly assigned to the knockout (Neat ${ }^{-/-}$) and wild-type control $\left(N e a t 1^{+/+}\right)$groups. Equal or nearly equal numbers of males and females were used to generate experimental cohorts. All animals were labelled with ear tags and experimenters who conducted tests and analysed results were blinded to their group allocation. To minimise the between-tests interference, evaluation of anxiety-like behaviour was performed first followed by activity and memory tests. Assessment of social behaviour was conducted at the end of the test battery as the most stressful paradigm. The Y-maze test was conducted in a procedure room different from that where locomotor activity had been tested to exclude interaction with surrounding cues. The resting time between tests was at least 7 days and the order of tests was the same for all mice.

\section{Statistical analysis}

Statistical analysis was performed using GraphPad Prism 6 and STATISTICA 12 software. In all cases, results are presented as the mean $\pm \mathrm{SE}$, with individual data shown where appropriate, and $n$ indicates the number of biological replicates. Homogeneity of variance was tested by Levene's test before ANOVA. Sample sizes were estimated based on the previous experience and calculation to achieve adequate power. Effect size was calculated using Cohen's $d$. Statistical details for each dataset are presented in the text and figure/table legends.

\section{Results}

\section{Predominantly non-neuronal expression of Neat 1 in the} murine CNS

Neat1 levels in the murine organs and tissues, as studied by RNA in situ hybridisation, vary significantly, with high levels in the digestive tract, medium - in lung and kidney, and low-in skeletal muscle and brain ${ }^{14}$. However, the distribution of Neat1 within anatomical structures in the brain and its relative expression in different cell types in the CNS are still largely unknown. To address this, we used RNAscope ${ }^{\circledast}$ ISH with $5^{\prime}$ end specific Neat1 probe in the sagittal brain sections as well as in the spinal cord of adult (2-month old) wild-type $\left(\mathrm{Neat}^{+/+}\right)$mice. As a negative control, we included brain sections from Neat1 knockout $\left(\mathrm{Neat}^{-/-}\right)$mice. This analysis revealed the expression of Neat1 throughout the brain and spinal cord (Fig. 1a). Region-wise, the highest Neat1 level was detected in corpus callosum, fornix and glia limitans, which are the brain structures mainly composed of glial cells. In neuron-dense areas, such as hippocampus and granule cell layer of the cerebellum, Neat1 was much less abundant and present in a dotted pattern (Fig. 1a, insets-hip and cer, respectively). In contrast, in many non-neuronal cells, the entire nucleus was darkly stained, as was especially obvious in the corpus callosum (Fig. 1a, inset-cc). Neat1 ISH also resulted in the staining of the cytoplasmic perinuclear region in a population of cells expressing highest Neat1 levels, which was attributed to a staining artefact (Fig. 1a, $c c$-arrowheads). In Neat $1^{-/-}$mice, only traces of Neat1 were detected in all anatomical brain regions except the cerebellum, where Neat1 signal was readily detectable in the granule cell layer (Fig. 1b). Similar to the brain, Neat1 signal was evenly distributed throughout the spinal cord of Neat $1^{+/+}$mice (Fig. 1c). To identify types of cells expressing Neat1, we used counterstaining of brain and spinal cord sections with Cresyl Violet (Nissl stain) after the RNAscope ${ }^{\circledR}$ ISH. Neat1 signal was observed in virtually all cell nuclei, with weak signal (few dots per cell) in cells with lightly stained nuclei, which were identified as neuronal (Fig. 1d), and strong signal in smaller nuclei often adjacent to neurons and corresponding to satellite cells-usually astrocytes and oligodendrocytes $^{34}$ (Fig. 1d, arrowheads and inset). Of note, we were unable to achieve the typical Nissl staining pattern (i.e., staining of both nuclei and cytoplasm of neurons) after RNAscope ${ }^{\circledR}$ ISH, which could be due to the ISH processing protocol.

Therefore, Neat1 is expressed throughout the mammalian CNS, predominantly in glial cells (astrocytes and oligodendrocytes), and is present at low levels in neurons.

\section{Neat1 knockout mice demonstrate abnormal behavioural response to stress}

Consistent with the original study ${ }^{35}$, Neat $1^{-/-}$mice showed no obvious deficits in general health, physical characteristics, or basic sensorimotor functions compared to their wild-type $\left(N e a t 1^{+/+}\right)$littermates, except for reduced body weight (Fig. 2a). However, we noticed that, despite normal behaviour while being observed in the cage without intervention, Neat $1^{-/-}$mice exhibited a panic-like escape response and hyperlocomotion 

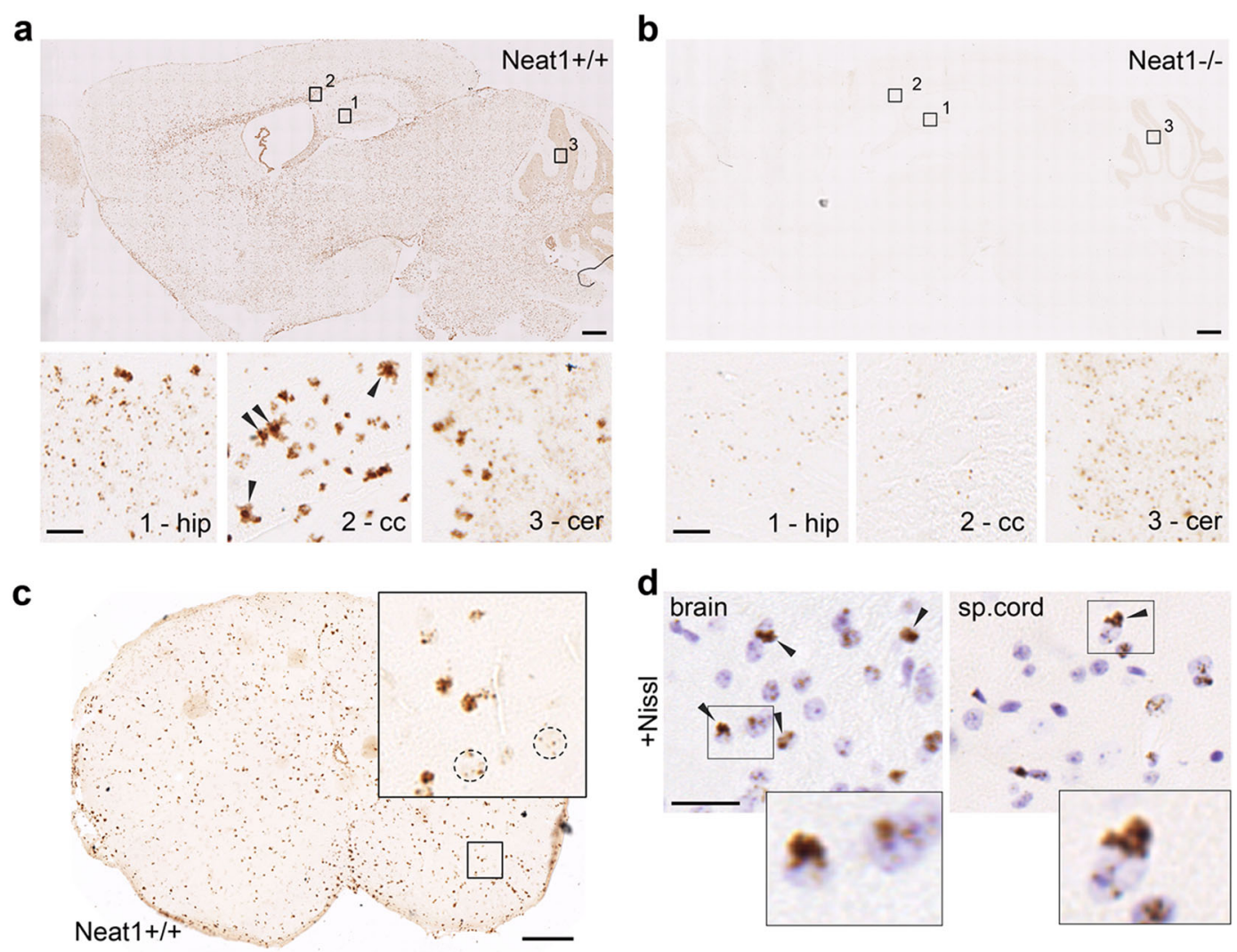

Fig. 1 Neat 1 is expressed in all cell types in the murine CNS. $\mathbf{a}$, b Neat1 is expressed at high levels in non-neuronal cells and low levels in neurons in the murine brain. Neat1 in situ hybridisation (RNAScope ${ }^{\otimes} \mid \mathrm{SH}$, with $5^{\prime}$ fragment Neat1 probe) on sagittal brain sections of Neat $1^{+/+}$(a) and Neat $1^{-/-}$(b) mice. Note low or absent Neat1 signal in all brain structures of Neat $1^{-1-}$ mice except the cerebellum (b). The bottom panels show insets from the top panels at higher magnification: 1-granular layer of the hippocampus (dentate gyrus); 2-corpus callosum; 3-Purkinje cells and granular layer of the cerebellum. Arrowheads indicate cells expressing high levels of Neat1 with characteristic perinuclear (cytoplasmic) reactivity (presumably, ISH artefact). c Neat1 ISH in the Neat $1^{+/+}$mouse spinal cord. Nuclei of motor neurons are circled. $\mathbf{d}$ Neat1 ISH with Nissl counterstain in the brain and spinal cord sections of a Neat $1^{+/+}$ mouse; arrowheads indicate satellite cells. Scale bars: $\mathbf{a}, \mathbf{b}$ - $200 \mu \mathrm{m}$ (main) and $20 \mu \mathrm{m}$ (insets); c - $100 \mu \mathrm{m}$;

d- $-35 \mu \mathrm{m}$.

(excessive running and jumping) upon contact with an experimenter, e.g., handling or cage changes (Supplementary Video S1). This phenotype gradually developed after weaning, and was apparent in 2-month-old animals. We generated experimental groups of littermate Neat $^{-/-}$ and $N e a t 1^{+/+}(n=9$ and 11 , respectively), that included both male and female mice, for behavioural analysis. Of note, the mouse strain used ${ }^{35}$ retains residual Neat1 expression, as has been shown in the original study and in our studies using RNA in situ hybridisation (Fig. 1) and RNA expression analysis (see below). However, to avoid ambiguity, we followed the original terminology for this mouse line and referred to these mice as "knockout" or "Neat $1^{-/-"}$ animals.

During the period spanning from the age of 3-7 months, the above experimental cohorts were assessed using a panel of behavioural tests. First, we attempted to examine motor function in these cohorts using rotarod, however it was not possible due to multiple escape attempts of $\mathrm{Neat1}^{-1-}$ mice after placing them on the apparatus. To assess the anxiety levels, we carried out the elevated plus maze (EPM) test. Neat $1^{-1-}$ mice presented with decreased anxiety-like behaviour in this test, as evidenced by a significantly higher number of entries to and increased time spent in open arms (Fig. 2b, c). However, an increase in the above readouts could be also interpreted as a behavioural phenotype of impulsivity ${ }^{36,37}$ and/ or high escape motivation ${ }^{38,39}$. Consistent with the latter interpretation, the number of head dips from the open arm towards the floor was significantly increased, whilst the number of stretch-attend postures, when the rodent remains motionless with its body stretched forward, was decreased for Neat $1^{-1-}$ mice (Fig. 2d, e). This is indicative of escape attempts and inadequate risk assessment ${ }^{37,40}$. There was no difference in the overall locomotor activity between the genotypes measured as the total number of arm entries (Fig. 2f), suggesting normal motor function in Neat $1^{-/-}$mice. 
a

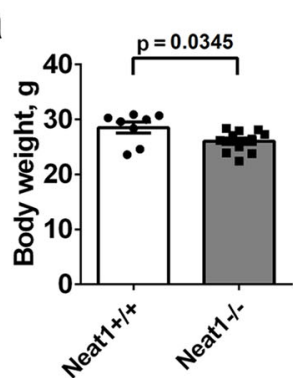

d

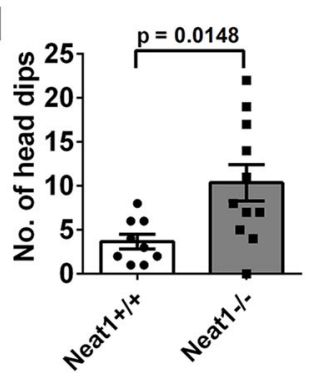

$g_{1250}$

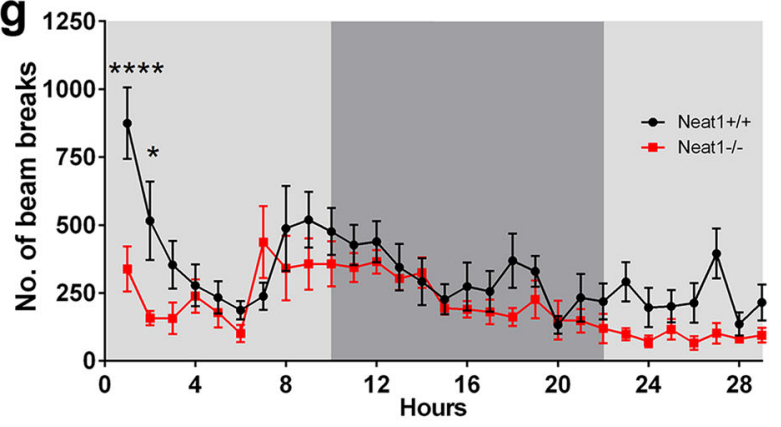

i

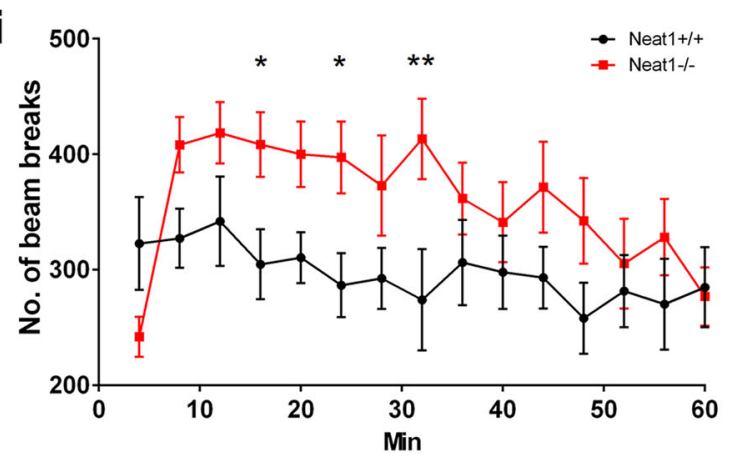

k

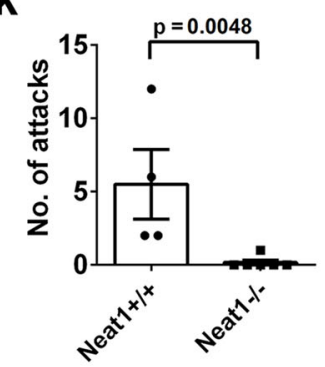

b

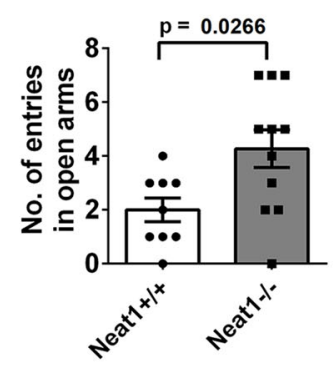

e

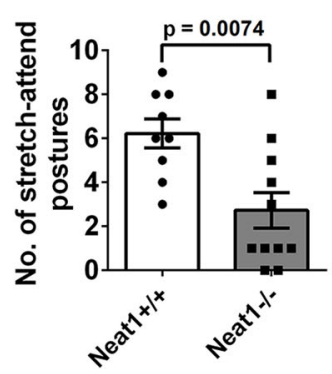

I

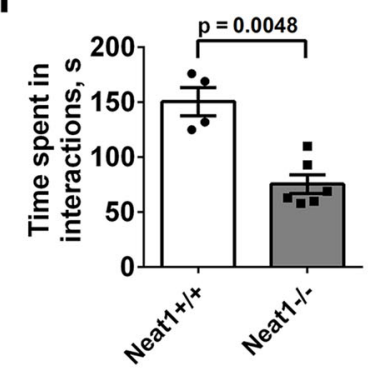

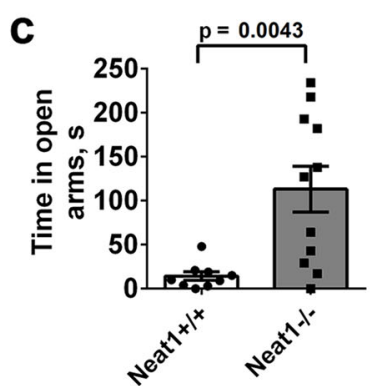

f
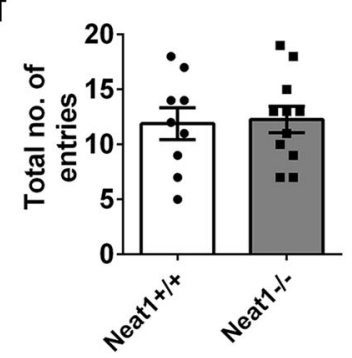

h
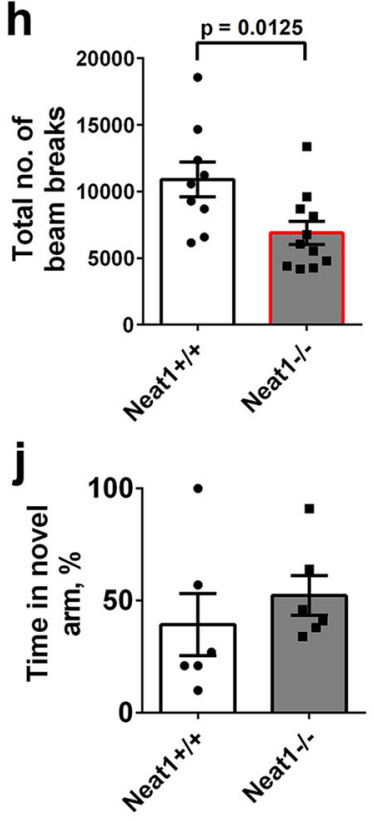

m

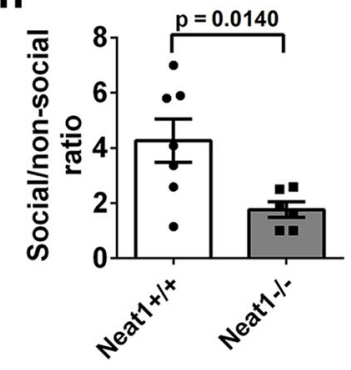

Fig. 2 (See legend on next page.) 
(see figure on previous page)

Fig. 2 Behavioural alterations in Neat $1^{-1-}$ mice. a Decreased body weight in Neat $1^{-1-}$ mice (Mann-Whitney U-test, effect size $=1.10 ;$ Neat ${ }^{-1-}$ $n=8$, Neat $\left.1^{+/+} n=10\right)$. b-f Increased number of entries (b) and time spent in the open arms (c) as well as increased number of head dips (d) and decreased number of stretch-attend postures (e) for Neat ${ }^{-1-}$ mice in elevated plus maze (EPM) (Mann-Whitney U-test, effect size $=1.13,1.54,1.26$, 1.42, respectively; Neat ${ }^{-1-} n=11$, Neat $1^{+/+} n=9$ ). Total number of arm entries was equal for the two genotypes $(\mathbf{f})$. g, $\mathbf{h}$ Reduced locomotor activity of $\mathrm{Neat1}^{-1-}$ mice in the Home Cage test (dim light conditions). Number of breaks per hour ( $\mathbf{g}$ ) and total number of breaks over the 30-h period $(\mathbf{h})$ are shown (two-way ANOVA with Sidak's multiple comparisons test in $(\mathbf{g}),{ }^{* * * *} p<0.0001,{ }^{*} p<0.05$, main effect of group $F(1,522)=38.90$, effect size $=3.03$; Mann-Whitney $U$ test in $(\mathbf{h})$, effect size $=1.32$; Neat $1^{-/-} n=10$, Neat ${ }^{+/+} n=9$ ). i Elevated locomotor activity of Neat $1^{-/-}$mice under bright light conditions (activity chamber) (two-way ANOVA with Uncorrected Fisher's LSD test, ${ }^{*} p<0.05$, ** $p<0.01$, main effect of group $F(1$, $261)=26.58$, effect size $=2.44$; Neat $1^{-1-} n=11$, Neat $7^{+/+} n=9$ ). $\mathbf{j}$ Lack of working memory deficits in Neat $1^{-1-}$ mice. Intact short-term memory in Neat $1^{-1-}$ mice in the $\mathrm{Y}$-maze test as indicated by the same percent of entries to novel arm $(\mathbf{k})$, as compared to Neat $1^{+/+}$mice (Mann-Whitney $U$-test; Neat1 $^{-/-} n=6$, Neat1 $\left.^{+/+} n=6\right) . \mathbf{k}$, I Decreased social interaction and aggression in Neat $1^{-/-}$mice in the Resident-Intruder test as revealed by lack of attacks $(\mathbf{k})$ and decreased time spent interacting with the intruder (I) (Mann-Whitney U-test, effect size $=2.75$ for both variable; Neat ${ }^{-1-} n=6$, Neat $\left.1^{+/+} n=4\right)$. $\mathbf{m}$ Decreased social interest in Neat ${ }^{-/-}$mice revealed in the Social Odour test (Mann-Whitney $U$-test, effect size $=1.82 ; \mathrm{Neat}^{-/}$ $n=5$, Neati $\left.^{+/+} n=6\right)$.

To analyse the locomotor activity of $N e a t 1^{-1-}$ mice under conditions of normal housing, we recorded their ambulatory activity for $24 \mathrm{~h}$ in the home cage $(\mathrm{HC})$ test. Neat $1^{-/-}$mice were overall hypoactive, with significantly reduced total activity during the test, as compared to Neat ${ }^{+/+}$mice (Fig. 2g, h). Decreased locomotor activity in the absence of motor impairment could be interpreted as depression-like behaviour. Surprisingly, Neat $1^{-1-}$ mice displayed dramatically reduced activity, as compared to Neat ${ }^{+/+}$mice, in the first $2 \mathrm{~h}$ of the test, i.e., when the animals encountered novel conditions, suggestive of increased anxiety. We were initially puzzled by the discrepancies between locomotor activity and anxiety levels in $\mathrm{Neat1}^{-/-}$mice recorded in HC and EPM tests. However, there is one important difference between the two tests which is the brightness of light. HC test is conducted at 30-40 lux (during the day phase), which is comparable to the light intensity under normal housing conditions. In contrast, EPM test is carried out under the bright light conditions ( 400 lux). Thus in the EPM test, the mice were subjected to strong aversive stimulation-a combination of novelty and bright light, which could be the reason for abnormal behaviour of $\mathrm{Neat}^{-{ }^{-1}}$ mice. To test this directly, we measured locomotor activity under bright light conditions (600 lux) for $1 \mathrm{~h}$ in the activity cage apparatus. Neat $1^{-1-}$ mice were indeed hyperactive under these conditions (Fig. 2i). Therefore, in the absence of strong stress, Neat1 ${ }^{-/-}$mice demonstrate decreased or similar (after habituation to novelty) level of locomotor activity to that of $\mathrm{Neat}^{+/+}$mice. However, a strong stressful stimulus, such as bright light, is sufficient to induce hyperactivity, impulsive reactions and possibly increased anxiety in Neat $1^{-/}$mice. Therefore, strong aversive stimulation converts the behaviour of Neat $1^{-/-}$ mice from hypoactive to hyperactive. To rule out possible memory deficits which could affect EPM test performance, we examined working memory using Y-maze forced alternation task. This test did not reveal any differences between the genotypes (Fig. $2 \mathrm{j}$ and data not shown).
To assess social behaviour of Neat $1^{-/-}$mice, we used Resident-Intruder test. In this test, $\mathrm{Neat}^{+/+}$mice demonstrated normal level of territorial defence behaviour, including moderately aggressive acts, such as lateral threats, chase and clinch attacks. In contrast, Neat $1^{-1-}$ mice showed nearly complete absence of aggression (Fig. 2k). In addition, Neat $1^{-/-}$mice had decreased physical interactions with the intruder (Fig. 2l). Altered social behaviour was further confirmed in the Social Odour test, where $\mathrm{Neat}^{-/-}$mice showed less interest in a social odour as compared to Neat ${ }^{+/+}$mice (Fig. $2 \mathrm{~m}$ ).

We next asked whether the phenotype in Neat $1^{-1-}$ mice would change during aging. Two key tests, EPM and HC, were performed in a group of 18-month-old mice (novel cohorts of animals not subjected to behavioural testing before). In the EPM test, aged Neat1 ${ }^{-/-}$mice showed behavioural patterns similar to the young animals, in that they also displayed preference for open arms and had increased number of head dips (Supplementary Fig. S2a-d). Interestingly, Neat $1^{-1-}$ mice were also hyperactive in this test, as indicated by increased total number of entries (Supplementary Fig. S2e). In the HC test, Neat $1^{-/-}$mice demonstrated reduced activity in the first $4 \mathrm{~h}$ (statistical significance reached for the first hour), however their total activity was similar to that of $\mathrm{Neat}^{+/+}$mice (Supplementary Fig. S2f, g). We also noticed an abnormal pattern of rhythmic fluctuations of activity in old Neat $1^{-1-}$ mice in this test: peaks of activity for Neat $1^{-/-}$mice were often in the reverse phase to those of $\mathrm{Neat}^{+/+}$animals (Supplementary Fig. S2f). Young Neat $1^{-1-}$ mice also demonstrated smoothened rhythmic activity pattern after habituation (from $8 \mathrm{~h}$ onward), lacking bursts of activity alternating with quiet periods typical for $\mathrm{Neat}^{+/+}$mice (Fig. $2 \mathrm{~g}$ ).

To summarise, Neat $1^{-1-}$ mice display a distinct behavioural phenotype manifested in stress-induced hyperlocomotion, decreased anxiety, disinhibition, altered daily rhythms of activity and impaired sociability, without concomitant working memory or motor deficits, and this phenotype is largely preserved during aging. 
Neat1 knockout mice do not present with neuronal loss, neuroinflammation or synaptic pathology

We next sought to understand the mechanisms underlying the observed behavioural phenotype in Neat $1^{-1-}$ mice. In the original study, Neat1 transcripts were not detected in E9.5 mouse embryos ${ }^{14}$. Similarly, using RNAScope ${ }^{\circledast}$ ISH, almost zero Neat1 expression was observed in the brain of E12.5 mouse embryos (Supplementary Fig. S3). This suggested that behavioural deficits in Neat $1^{-/-}$mice are unlikely to be caused by developmental defects. Analysis of the gross morphology of the brain of adult $\mathrm{Neat1}^{-/-}$and Neat1 ${ }^{+/+}$mice using Nissl staining and NeuN immunohistochemistry did not reveal differences between the genotypes (Fig. 3a, b). Neuronal numbers in $\mathrm{Neat}^{-1-}$ mice were normal at least in the frontal cortex, consistent with the absence of overt neurological symptoms (Fig. 3c). However, we detected a small but significant decrease in the brain weight of
Neat $^{-1-}$ mice (Fig. 3d), which was in line with their reduced body weight (Fig. 2a).

We next examined levels of several markers commonly altered in models of neurological diseases. Neuroinflammation is typical for many neurological conditions, and Neat1 itself has been implicated in immune response $^{7,41,42}$. However immunohistochemistry and western blotting did not reveal any signs of astrogliosis or microgliosis in the brain of Neat1 $1^{-1-}$ mice (Fig. 3e, f). Synapse dysfunction is a major determinant of many neurological diseases. However distribution and levels of the two critical synaptic proteins, synaptophysin and PSD95 (encoded by Dlg4 gene) in the brain of Neat $1^{-/}$ mice appeared normal (Fig. 3g, h).

Therefore, behavioural deficits in Neat1 $1^{-1-}$ mice cannot be explained by neuronal loss, neuroinflammatory phenotype or gross synaptic dysfunction.

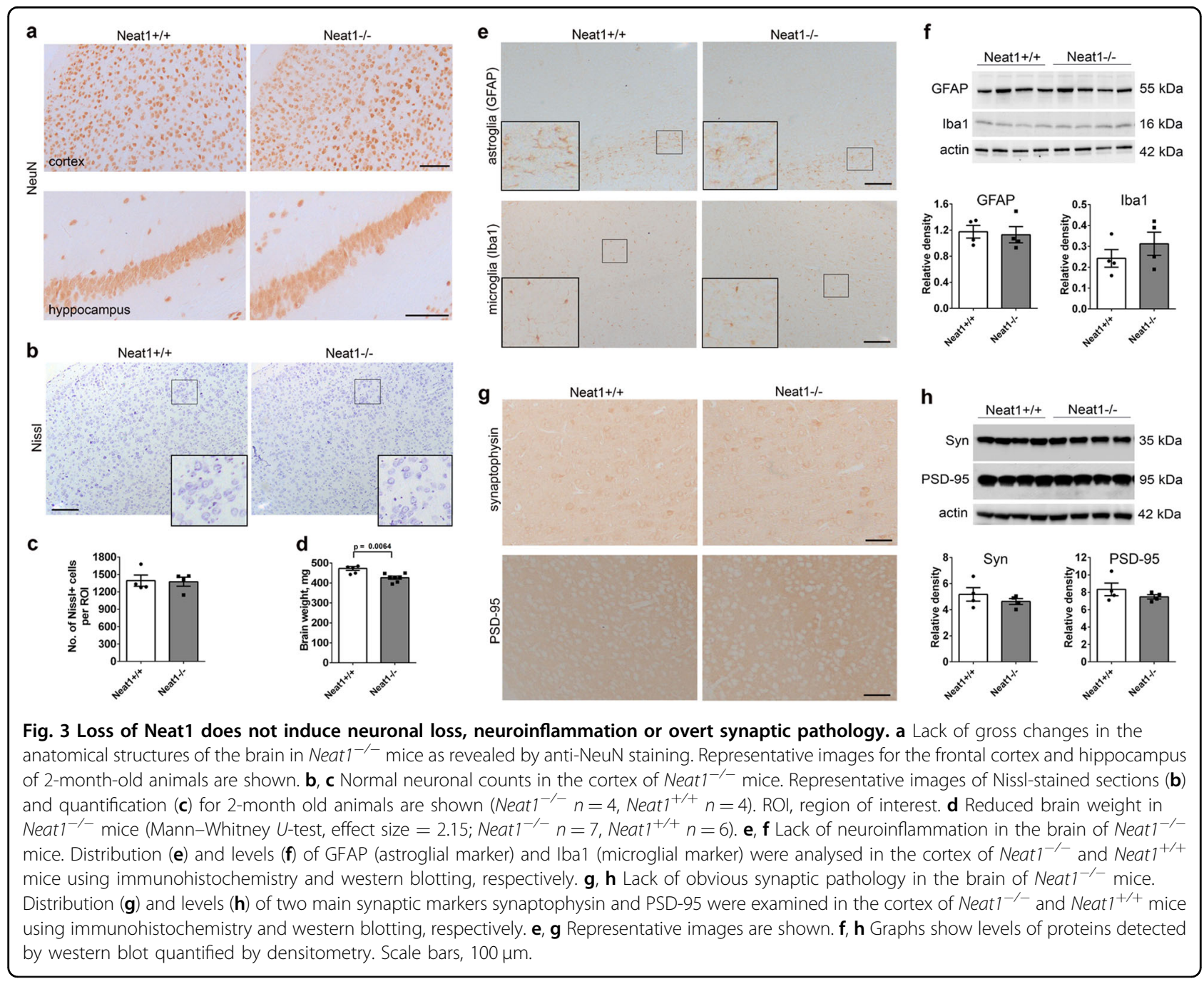


Neat $1^{-/-}$neurons have altered calcium homoeostasis and are hyperexcitable in vitro and in vivo

We speculated that abnormal behavioural responses of Neat $1^{-/-}$mice may be determined by intrinsic changes in neurons, e.g., their altered excitability. It has been shown previously that human iPSC-derived neurons become hyperexcitable after acute downregulation of NEAT $1^{13}$. We prepared and analysed primary cultures of the hippocampal neurons from newborn $\mathrm{Neat}^{+/+}$and $\mathrm{Neat}^{-/-}$ mice. Morphological analysis of neurons transiently transfected to express GFP did not reveal significant differences in the neurite outgrowth and branching between the genotypes (Fig. 4a). To assess excitability properties of these neurons, including transmembrane currents and the ability to generate induced action potentials (iAPs), we employed patch-clamp whole-cell electrophysiology. In current-clamp studies, $50 \%$ of $\mathrm{Neat1}^{-/-}$neurons demonstrated spontaneous action potentials, whereas in
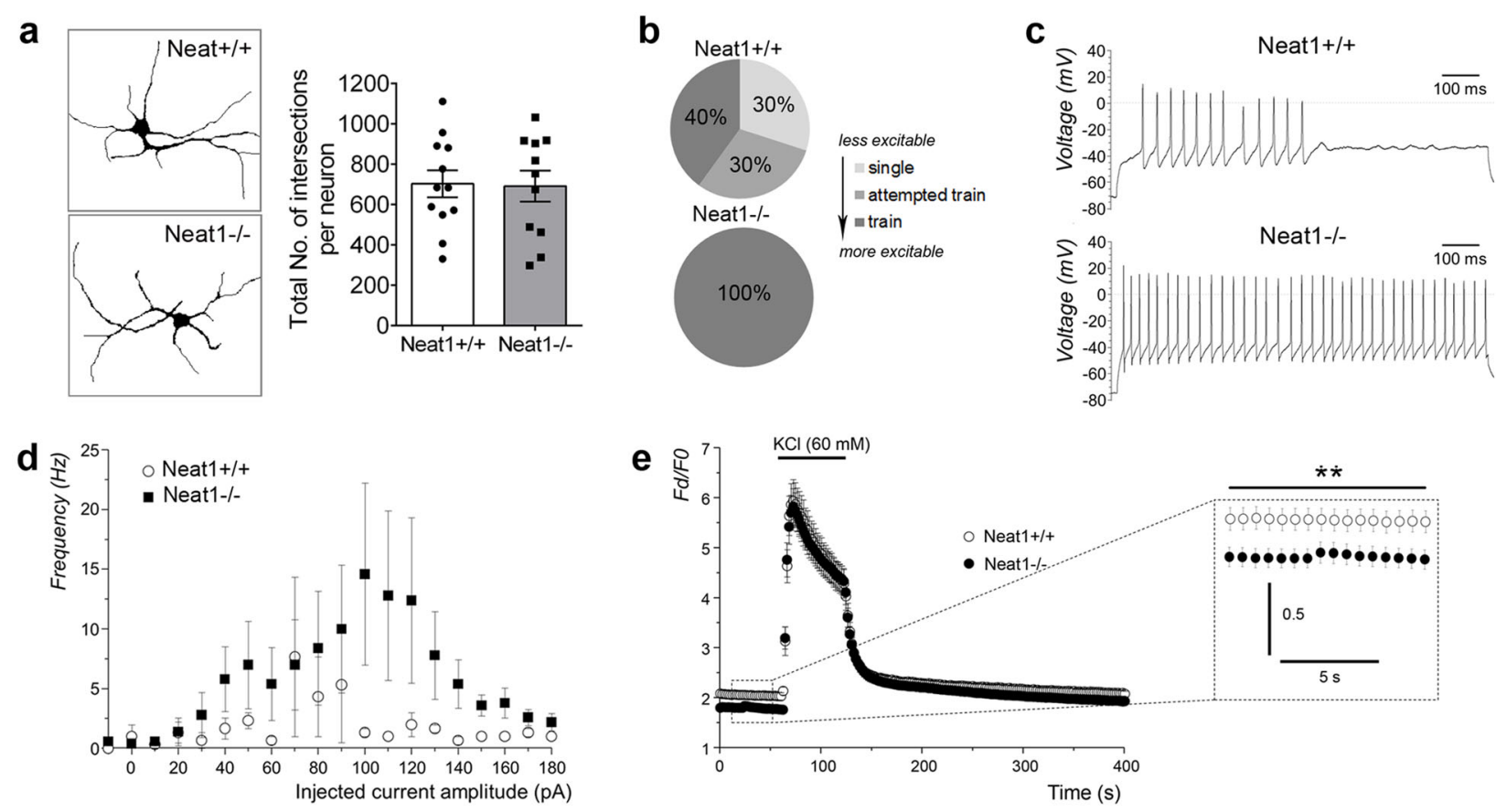

e

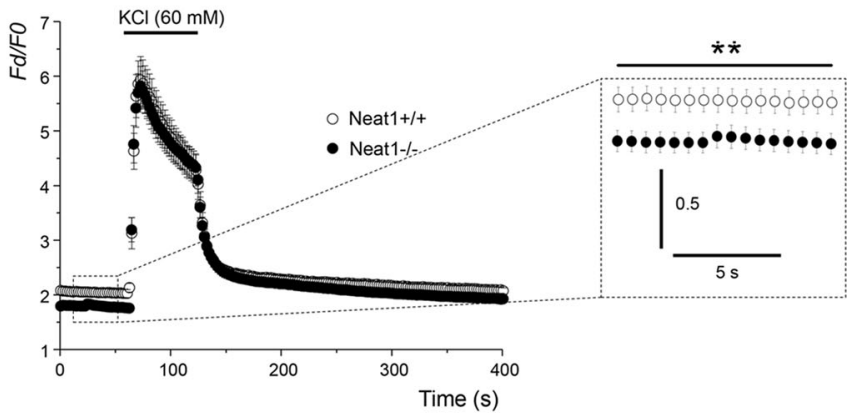

$\mathbf{f}$
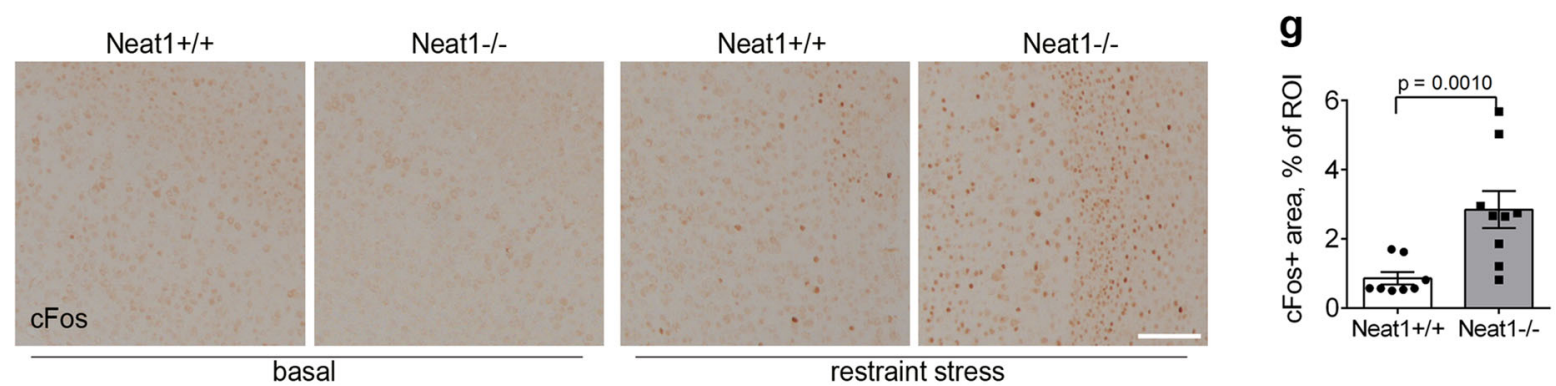

Fig. 4 Neat $1^{-/-}$neurons are intrinsically hyperexcitable. a Primary hippocampal neurons isolated from Neat $1^{-/-}$and Neat $1^{+/+}$newborn mice do not display differences in cell morphology and neurite branching. Traces (left) and branching analysis (right) for DIV7 neurons are shown (Neat ${ }^{-1-}$ $n=12$, Neat $1^{+/+} n=12$ ). Neurons were transiently transfected to express GFP and analysed $24 \mathrm{~h}$ post-transfection. $\mathbf{b}$, $\mathbf{c}$ All Neat $1^{-1-}$ neurons generate trains of induced action potentials (iAPs). The proportion of neurons able to generate three patterns of induced activity (single action potential, attempted trains of action potentials or trains of action potentials) is shown (Neat1 ${ }^{-/} n=4$, Neat $^{+/+} n=7$ ) (b). Exemplar traces of current-clamp recordings when Neat $1^{+/+}$(upper panel) and Neat $1^{-1-}$ (lower panel) neurons were held at between -70 and $-80 \mathrm{mV}$ and a following 1-s step of injected currents to the value that produced maximal trains of iAPs are also shown (c). $\mathbf{d}$ Representative spike frequency plots for Neat $1^{+/+}$(open circles) and Neat $1^{-/-}$(filled squares) neurons exhibiting trains of iAPs. e KCl-induced $\mathrm{Ca}^{2+}$ response in Neat $1^{-1-}$ and Neat $1^{+/+}$ neurons (two-way ANOVA with Fisher's LSD test, ${ }^{* *} p<0.01$, main effect of group $F(1,1440)=255.0$, effect size $=4.82,{ }^{* *} p<0.01 ; N e a t 1^{-/-} n=27$, Neat $\left.7^{+/+} n=20\right)$. Inset shows the difference in the basal $\mathrm{Ca}^{2+}$ levels between the phenotypes. $\mathbf{f}, \mathbf{g}$ Increased number of cFos-positive neurons in the cortex of Neat ${ }^{-1-}$ mice after the restraint stress (immobilisation). Representative images (f) and quantification (g) are shown (Mann-Whitney U-test, effect size $=2.25$; Neat $^{-1-} n=3$, Neat $1^{+/+} n=3 ; 9$ ROls per group). ROI, region of interest. Scale bar, $100 \mu \mathrm{m}$. 
the $\mathrm{Neat1}^{+/+}$group, only $36 \%$ neurons were spontaneously active (Supplementary Table S1). All examined Neat $^{-1-}$ neurons displayed repetitive induced action potentials (iAPs "trains") vs. only $40 \%$ of the neurons from the Neat $1^{+/+}$group (Fig. 4b, c; Supplementary Table S2). Another evidence of augmented activity of Neat $1^{-/-}$ neurons was that they demonstrated significantly higher frequency of train iAPs compared to Neat $1^{+/+}$neurons (Fig. 4d). Interestingly, while having similar values of the resting membrane potential, Neat1 ${ }^{-/-}$neurons had significantly faster depolarisation and repolarisation rates $(103.1 \pm 18.4 \mathrm{~V} / \mathrm{s}$ and $-71.6 \pm 14.1 \mathrm{~V} / \mathrm{s}$ vs. $61.6 \pm 9.6 \mathrm{~V} / \mathrm{s}$ and $-37.9 \pm 8.1 \mathrm{~V} / \mathrm{s}$ for Neat $1^{-/-}$and Neat $1^{+/+}$neurons, respectively; $p<0.05$ ).

To understand the basis of the enhanced excitability of Neat $1^{-1-}$ neurons, transmembrane voltage-gated $\mathrm{Na}^{+}$ and $\mathrm{K}^{+}$currents were measured using voltage-clamp mode. Neat $1^{-1-}$ neurons had increased densities for both inward and outward transmembrane currents with significant difference from Neat $1^{+/+}$neurons for $\mathrm{Na}^{+}(p<$ 0.05 ) but not for $\mathrm{K}^{+}$voltage-gated currents (Supplementary Fig. S4a, b; Supplementary Table S3). Activation/ inactivation profiles of the voltage-activated $\mathrm{Na}^{+}$currents for Neat $^{-/-}$neurons exhibited larger availability windows with high G/Gmax maxima and twice as much difference between mean half-activation and halfinhibition voltages (Va50-Vi50) $5.0 \pm 1.0 \mathrm{mV}$ vs. the Neat $^{+/+}$group $2.9 \pm 1.1 \mathrm{mV}$. Vm values of both Neat $1^{-/-}$ and $\mathrm{Neat}^{+/+}$neurons were falling within those windows however the proportion of spontaneously active neurons was higher in the Neat $1^{-/-}$group (Supplementary Fig. S4c, d; Supplementary Table S1). The above data suggest that increased excitability of Neat $1^{-/-}$neurons is determined by the increased transmembrane ion conductances particularly through $\mathrm{Na}^{+}$voltage-gated channels, which are responsible for the ascending phase of neuronal action potentials.

To explore how the changes in excitability of the Neat $1^{-1-}$ neurons correspond to the regulation of calcium $\left(\mathrm{Ca}^{2+}\right)$ homoeostasis, we performed $\mathrm{Ca}^{2+}$ imaging experiments using ratiometric Fura-2 AM dye to examine the changes of the intracellular $\mathrm{Ca}^{2+}$ induced by $\mathrm{KCl}$. Although $\mathrm{Ca}^{2+}$ responses upon $\mathrm{KCl}(60 \mathrm{mM})$ application in the neurons of both genotypes were similar, we found that $N e a t 1^{-/-}$neurons have significantly lower $(p<$ 0.0001) basal levels of $\mathrm{Ca}^{2+}$ (Fig. 4e).

We next sought to confirm the increased activity of Neat $1^{-1-}$ neurons in vivo. The immediate early gene $c$ Fos is the most well-known molecular marker of neuronal activity $^{43}$. Expression of this gene can be experimentally induced in the rodent brain in vivo by certain stresses such as immobilisation (restraint stress) ${ }^{44}$. We subjected Neat $1^{-/-}$and Neat $1^{+/+}$mice to $60 \mathrm{~min}$ restraint stress followed by $60 \mathrm{~min}$ recovery and subsequently analysed
cFos protein expression in the brain of these mice by immunohistochemistry. We found that the number of cFos-positive cells in the cortex of stressed $\mathrm{Neat}^{-/-}$mice was significantly higher as compared to stressed $\mathrm{Neat} \mathrm{t}^{+/+}$ mice (Fig. 4f, g).

Overall, our data indicate that $\mathrm{Neat1}^{-/-}$neurons are intrinsically hyperexcitable and have deficiencies in calcium homoeostasis, which can result in abnormal gene expression responses in vivo.

\section{NEAT1_2 induction in neurons is a late event during stress}

The mouse strain used in this study lacks the expression of both Neat1 isoforms, the constitutively expressed Neat1_1 and the inducible Neat1_2. So far, only one type of stress, pharmacologically-induced seizures, is known to induce de novo Neat1_2 synthesis in the brain ${ }^{45}$. However, whereas Neat1_1 upregulation was an early event in the above study ( $1 \mathrm{~h}$ post-stress), Neat1_2 induction/ expression was delayed, and its peak occurred $8 \mathrm{~h}$ poststress, with its levels still high $24 \mathrm{~h}$ post-stress. We asked whether the behaviour phenotype of Neat1 ${ }^{-1-}$ mice and the phenotypes detected in cultured neurons derived from these mice may be attributed to the loss of Neat1_2 induction. Firstly, we performed RNAScope ${ }^{\circledast}$ ISH on the brain of wild-type mice after restraint stress $(60 \mathrm{~min}$ stress/60 min recovery), using a Neat1_2 specific ISH probe, but did not detect any Neat1_2 signal (data not shown). Since we were unable to induce Neat1_2 expression in wild-type mouse primary neurons with any known stressors, we used a recently established stress paradigm $^{5}$ in human embryonic stem (ES) cell derived neuronal cultures. Day 36 neurons were subjected to an acute oxidative stress using $\mathrm{NaAsO} 2$ (sodium arsenite) for $1 \mathrm{~h}$ and left to recover for 1-48 h, or were subjected to 10fold lower $\mathrm{NaAsO} 2$ concentration for 24 h, and NEAT1_2 expression was examined by RNA-FISH. Human ES cell derived neurons did not have detectable NEAT1_2 expression under basal conditions, immediately after stress and early during recovery (Fig. 5a). NEAT1_2 signal started to appear after $6 \mathrm{~h}$ of recovery, with a peak at $24 \mathrm{~h}$ of recovery, and was still detectable after $48 \mathrm{~h}$ of recovery (Fig. 5a). NEAT1_2 induction was also confirmed by qRTPCR (Fig. 5b). Prolonged treatment with low NaAsO2 concentration was not as efficient in NEAT1_2 induction (Fig. 5a). Another stressor known to robustly trigger NEAT1_2 expression in stable cell lines, a proteasome inhibitor MG132 ${ }^{10}$, also induced NEAT1_2 expression, albeit to a lesser extent than $\mathrm{NaAsO} 2$ (Fig. 5a).

These in vitro data suggest that expression of NEAT1_2 is a late event during stress and that induction of Neat1_2 expression is unlikely to mediate the phenotypes detected in cultured mouse Neat $1^{-/-}$neurons and behaviour responses in $\mathrm{Neat}^{-/-}$mice. 


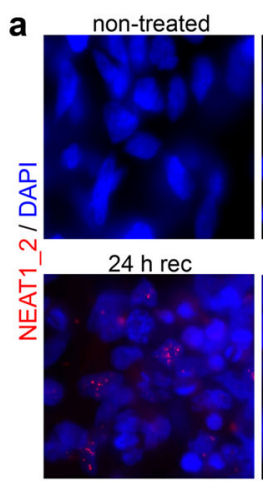

non-treated

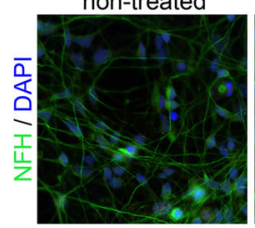

$1 \mathrm{~h} \mathrm{NaAsO} 2$

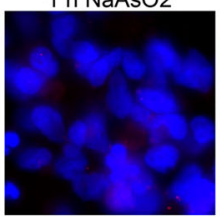

$48 \mathrm{~h} \mathrm{rec}$

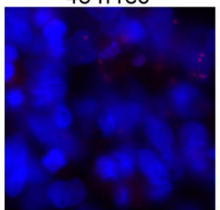

$3 \mathrm{~h} \mathrm{rec}$

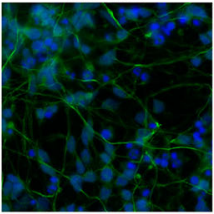

$3 \mathrm{~h}$ rec
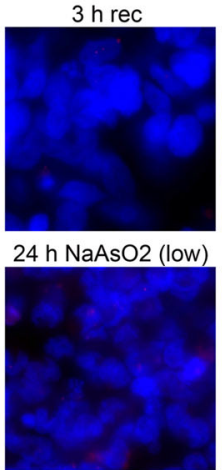

$6 \mathrm{~h}$ rec

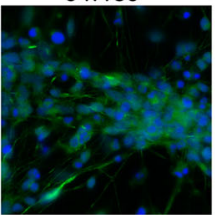

$6 \mathrm{~h}$ rec

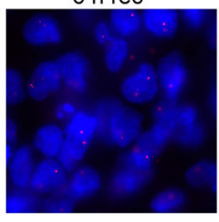

6 h MG132

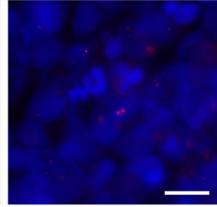

$24 \mathrm{~h} \mathrm{NaAsO} 2$ (low) b

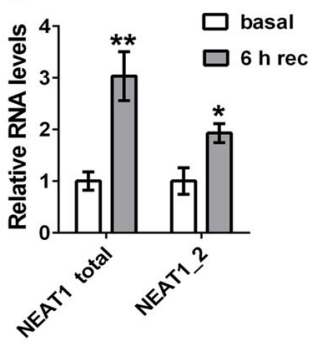

Fig. 5 Stress-induced NEAT1_2 induction in neurons is a late event during stress. a De novo synthesis of NEAT1_2 in human ES cell derived neurons occurs late during the recovery from oxidative stress. Day 36 neurons were treated with $0.5 \mathrm{mM} \mathrm{NaAsO} 2$ for $1 \overline{\mathrm{h}}$ and left to recover for the indicated periods of time; or treated with $0.05 \mathrm{mM} \mathrm{NaAsO} 2$ ("low" concentration) for $24 \mathrm{~h}$; or treated with $10 \mu \mathrm{M}$ MG132 for 6 h, and analysed by NEAT1_2 RNA-FISH. Bottom panel shows neurite network integrity at different stages of recovery (detected using anti-neurofilament H, NFH antibody). Representative images are shown. Scale bars, $20 \mu \mathrm{m}$ (top panels) and 50 $\mu \mathrm{m}$ (bottom panel). b Upregulated total NEAT1 and NEAT1_2 expression during the recovery from oxidative stress as detected by qRT-PCR. Neurons were treated with $0.5 \mathrm{mM}$ NaAsO2 for $1 \mathrm{~h}$ and left to recover for 6 h (Mann-Whitney $U$-test, $\left.{ }^{*} p<0.05,{ }^{* *} p<0.01, n=8\right)$.

\section{Loss of Neat 1 perturbs local chromosomal environment} and leads to subtle downregulation of multiple genes in the brain

Absence of detectable changes in the core CNS function-related markers (Fig. 3) suggested the existence of more subtle molecular changes that result in abnormal neuronal excitability and altered behavioural responses in Neat $1^{-/-}$mice. In order to identify possible changes of the gene expression profiles in the CNS of Neat $1^{-/-}$mice, we performed transcriptomic analysis of cortical tissue. Total RNA (RIN 8.7-9.2) from the whole cortices of 2month-old animals (four per genotype) was used. Prior to sequencing, Neat1 levels in these samples were measured by qRT-PCR. In the original study, total Neat1 (Neat1_1 + Neat1_2) levels in mouse embryonic fibroblasts were found to be decreased to $6.0 \pm 0.5 \%$, whereas Neat1_2 was undetectable ${ }^{14}$. In the cortex of adult mice, total Neat1 was found to be decreased to $18.9 \pm 0.02 \%$, and Neat1_2 - to $8.2 \pm 0.01 \%$ (Fig. 6a), probably reflecting tissue- and developmental stage-specific differences in the efficiency of Neat1 silencing. Using Illumina NextSeq500 platform and paired-end sequencing, we obtained $\sim 50$ million reads per sample. One of the $\mathrm{Neat}^{+/+}$samples showed abnormally low Neat1 expression and was identified as an outlier in the principal component analysis leading to its exclusion from the differential gene expression analysis. Examination of RNA-Seq peaks revealed that Neat1_1 is the main transcript expressed in the cortex, while Neat1_2 levels are very low in this tissue, consistent with previous reports ${ }^{14,45}$. In accord with qRTPCR data, RNA-Seq analysis detected significantly reduced yet still detectable levels of Neat1 in the cortex of Neat $1^{-/-}$mice (Fig. 6b, c).

Differential gene expression analysis showed very subtle changes in the gene expression in $\mathrm{Neat}^{-/-}$mice, consistent with the lack of overt neurological deficiencies in these mice. Only 14 genes were found to be significantly changed (adjusted $p$-value, padj < 0.05), including ten genes with known function (Cd6, Neat1, Rprm, Col62a, Prune2, Frmd8, Eps8l1, Crip2, Carnmt1, Snhg1, Supplementary Table S4). We next used the list of genes with at least 1.5-fold change in their expression (non-adjusted $p$-value $<0.01$ ), which was met by 289 genes, to assess gross gene expression changes. Overwhelmingly more genes from this list were found to be downregulated (260: 89.3\%) than upregulated (29: 10.7\%) (Fig. 6d, e), indicating that Neat1 positively regulates gene expression of a large subset of genes. Puzzled by this finding, we additionally performed a low coverage RNA-Seq analysis of another cohort of $\mathrm{Neat1}^{-/-}$and Neat1 ${ }^{+/+}$animals (aged 1 month). Similar to the 2-month-old mice, changes in the gene expression were very subtle, however, the vast majority (85\%) of differentially expressed genes (DEGs: $>1.5$ fold change, $p$-value $<0.05$ ) were also found to be downregulated. In this latter mouse cohort, only four genes showed significantly altered expression (padj < 0.05), 


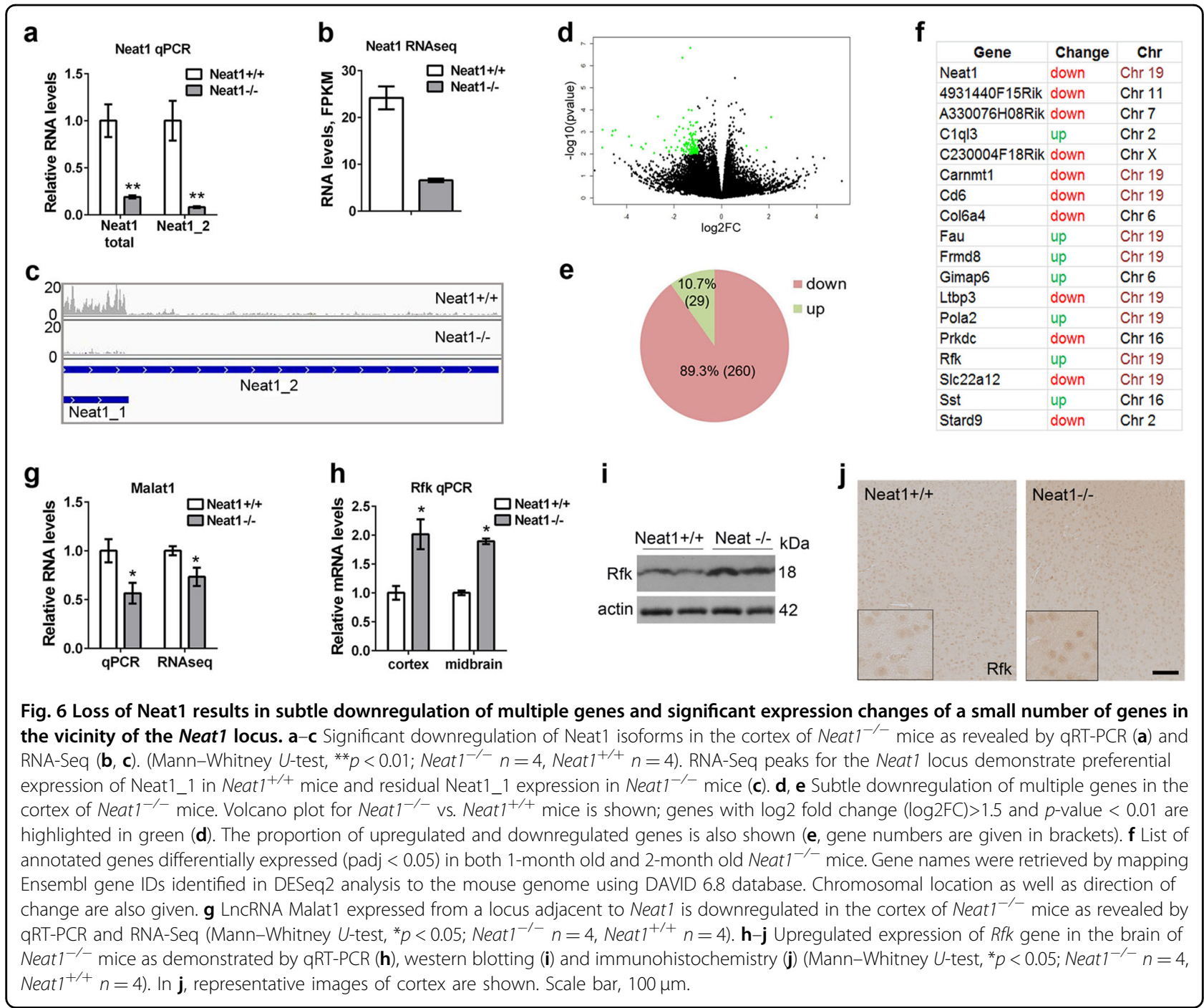

namely Neat1, Frmd8, Carnmt1 and Malat1. We compared the lists of genes whose expression was changed $>1.5$ fold in the cohorts of 1-month old mice (cut-off $p$ value $<0.05, n=474$ ) and 2-month-old mice (cut-off $p$-value $<0.01, n=289$ ). Altogether, 29 genes, 18 of which are genes with known function, were found to appear in both groups, including previously identified genes with robust changes-Neat1, Frmd8 and Cd6 (Fig. 6f). Positional analysis for these genes showed that half of them $(9 / 18)$ are located on chromosome 19, and some of them-in close vicinity to the Neat1 locus (Frmd8, Ltbp1, Pola2, Fau) (Fig. 6f). However, expression of other genes surrounding the Neat1 locus (Scyl1, Slc25a45, Tigd3, $D p f 2$, and Cdc42ep2) was not altered.

We next performed enrichment analysis on the set of genes with at least 1.5 fold change in their expression $(p-$ value $<0.01)$ for 2 -month-old mice. Out of these 289 mouse Ensembl gene IDs, 60 could be mapped to known genes using DAVID 6.8 tool. Small enrichment in anion transport genes (GO:0006820, $p=0.00678$ : Gabrr2, Anxa1, Kcnj10, Slc2a2 and Slc22a12) was identified, of which one gene, Gabrr2, attracted our attention. Stressinduced impulsive behaviours in animals may be suggestive of abnormal inhibitory neurotransmission, which is mediated by GABA receptors. We therefore examined the expression of Gabrr2 and two major GABA receptor subunit genes, Gabra1 and Gabra2 by qRT-PCR. Although Gabrr2 mRNA was indeed confirmed to be downregulated, Gabra1 and Gabra2 expression was unaltered (Supplementary Fig. S5a). Further, numbers and distribution of inhibitory Gad67-positive interneurons in the brain as well as levels of Gad67 in the cortex were not changed in Neat $1^{-/-}$mice (Supplementary Fig. S5b, c). Thus we concluded that GABAergic inhibitory transmission is unlikely to be significantly affected in Neat $1^{-/-}$ mice.

One gene found to be significantly changed (padj $<0.05$ ) in 1 -month Neat $^{-/-}$mice was Malat1. Malat1 is a 
conserved lncRNA transcribed from a locus adjacent to Neat 1 and highly expressed in the brain ${ }^{46}$. Malat 1 null mice have also been reported to have upregulated expression of several genes in close vicinity to the Malat1 locus, including Neat1 and Frmd8, in the liver and brain ${ }^{46}$. However, another Malat1 knockout mouse strain displayed decreased Neat1 expression in a subset of tissues, although brain was not examined ${ }^{47}$. Our RNA-Seq analysis showed Malat1 downregulation in Neat ${ }^{-/-}$mice, in both age groups analysed, and this result was confirmed by qRT-PCR (Fig. $6 \mathrm{~g}$ ).

Another gene found significantly upregulated in both 1-month and 2-month old Neat $1^{-/-}$mice encodes riboflavin kinase (Rfk) enzyme. Intriguingly, human riboflavin kinase has been recently linked to neurological diseases ${ }^{48}$ and regulation of circadian rhythms ${ }^{49}$. We confirmed that Rfk mRNA is $\sim 2$ fold upregulated in the cortex and midbrain of Neat $^{-/-}$mice by qRT-PCR (Fig. 6h). Consistent with this, Rfk protein levels were higher in the cortex of Neat1 ${ }^{-/-}$mice, as analysed by western blot (Fig. 6i). Immunohistochemical analysis revealed exclusively neuronal Rfk expression, and Rfk upregulation was also evident in the brain sections of Neat $1^{-/-}$mice (Fig. 6j).

In sum, Neat 1 acts as a weak positive modulator of gene expression in the brain, whilst its loss impacts on the transcription of a small pool of genes in the vicinity of the Neat1 locus.

\section{Loss of Neat1 affects alternative splicing of genes involved in neuronal homoeostasis}

RNA-Seq datasets for Neat $1^{-/-}$and $\mathrm{Neat1}^{+/+}$mice were further used to analyse possible changes in the alternative splicing in the brain of Neat1 ${ }^{-/-}$mice. For that, a recently developed pipeline, LeafCutter ${ }^{26}$, was utilised. Altogether, 14 differentially spliced genes were identified in Neat $1^{-1-}$ mice using a $<20 \%$ FDR threshold (Fig. 7a, b and Supplementary Table S5). All these genes are highly expressed in the brain and two of them-almost

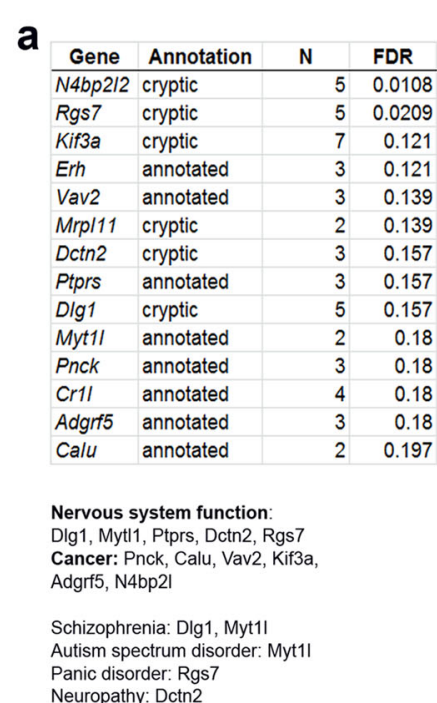

C

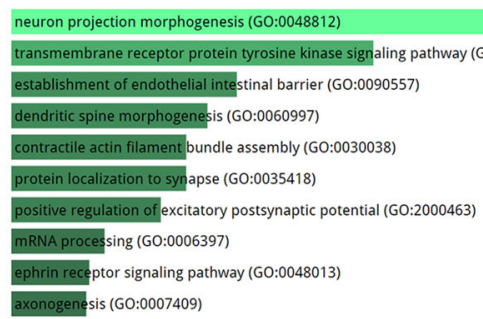

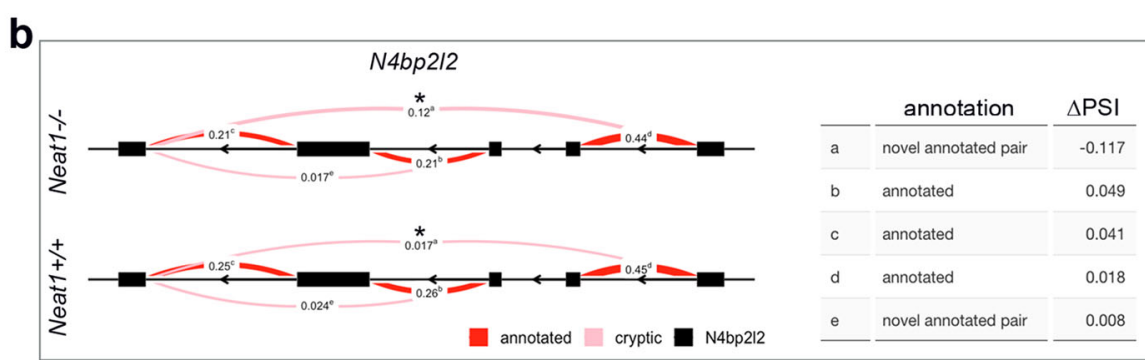

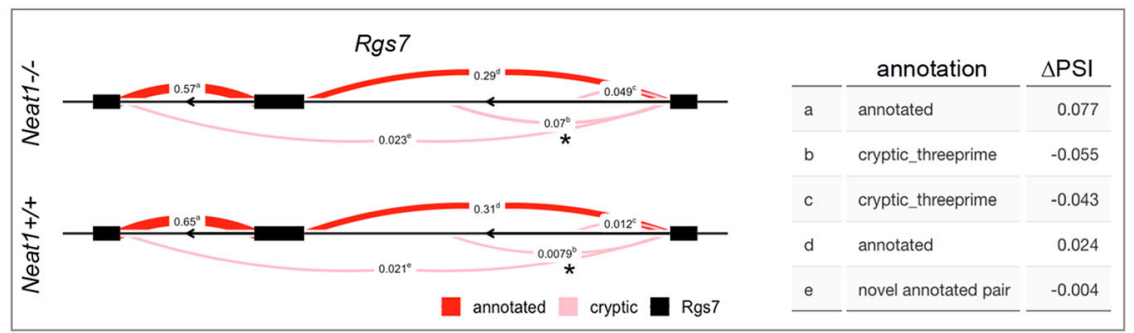

d

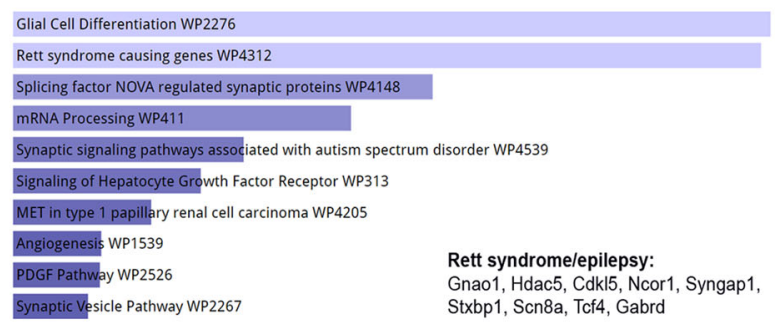

Fig. 7 Loss of Neat 1 causes changes in the alternative splicing of genes involved in synaptic function and RNA processing. a List of genes differentially spliced in the cortex of $\mathrm{Neat}^{-1-}$ mice. Functional association and role in disease for these genes are also given. $\mathrm{N}$ number of introns in a cluster, FDR false discovery rate; "annotated", all introns in the intron cluster are annotated in GENCODE release M20; "cryptic", at least one intron in the intron cluster is not annotated in GENCODE release M20. b Splicing diagrams showing differential intronic usage in two intron clusters of N4bp2/2

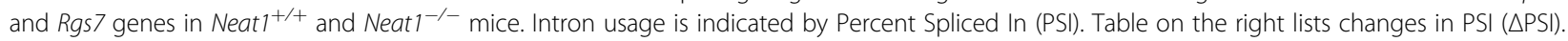
c, $\mathbf{d}$ Enrichment analysis of differentially spliced genes as analysed using Enrichr. GO term Biological Process (c) and Wiki Pathways (d), alongside with their functional association and role in disease are shown. 
exclusively in the brain (Myt1l, Rgs7). Most of these genes are also important for the CNS function, including those with roles in neuron proliferation, differentiation, cell-cell interaction, intraneuronal transport, synaptogenesis, primary axonogenesis, and axon guidance (Dlg1, Myt1l, Ptprs, Dctn2, Rgs7). Further, some genes are genetically linked to psychiatric disorders such as schizophrenia $(\text { Dlg1, Myt } 1 l)^{50,51}$, autism spectrum disorder $(M y t 1 l)^{52,53}$ and panic disorder $(\operatorname{Rgs} 7)^{54}$. Other genes from this list are linked to various types of cancer (Pnck, Calu, Vav2, Kif3a, Adgrf5, N4bp2l2).

For the enrichment/pathway analysis we used a $p$-value cut-off of $<0.05$ which was met by 671 genes (Supplementary Table S5). The use of a relaxed cut-off ( $p$-value instead of padj) in this case was possible because only truly positives are expected to cluster in functional categories whereas false positives should be functionally unrelated $^{55}$. Analysis of GO Biological process enriched terms using Enrichr revealed that a significant number of differentially spliced genes were involved in axonogenesis, protein localisation to synapse and dendritic morphogenesis, including genes critical for neuronal homoeostasis and implicated in neurological diseases, e.g., Shank1, Bdnf, Map2, Dlg4, Nrxn1, Nrxn2 and Ncam1 (Fig. 7c). Another enriched category was RNA processing, including genes encoding abundant RNA-binding proteins Cpsf6, Hnrnpk, Hnrnph1, Hnrnpa2b1, Hnrnpd, Srsf5 and Srsf7. Pathway analysis of this dataset also identified genes involved in synaptic function (e.g., Syngap1, Camk2b, Cacna1a, Cacna1d, Gria4) and mRNA processing (e.g., Celf1, Cstf2, Pcbp2, Hnrnpk, Hnrnph1, Hnrnpa $2 b 1$, alongside with several genes encoding serine and arginine splicing factors) (Fig. 7d). Multiple genes from this list are linked to neurological conditions. Calcium channel subunits Cacnald and Cacnala are associated with autism spectrum disorders ${ }^{56}$ and with familial hemiplegic migraine and episodic ataxia ${ }^{57,58}$, respectively. Variants in the Gria4 gene are linked to schizophrenia and intellectual disability ${ }^{5-61}$. Consistent with recently reported dysregulation of NEAT1 in epilepsy ${ }^{13,45,62}$, the second enriched pathway in our analysis was "Rett syndrome causing genes" which contained 9 genes implicated in epilepsy, such as Gabrd, Gnao1, Stxbp1 and Scn8a.

Overall, we showed that loss of Neat1 perturbs alternative splicing in the brain, with the most significant effect on the genes related to synaptic function and RNA metabolism and implicated in neurological diseases.

\section{Discussion}

LncRNAs play an important role in fine-tuning the functions specific for different cell types, and their activity might be especially important in the adult nervous system. Indeed, IncRNAs have been reported to regulate critical processes in the developing and adult brain, including cell differentiation, neurite elaboration and synaptogenesis ${ }^{63,64}$. NEAT1 is an abundant lncRNA known to be expressed in the mammalian CNS however its role in the CNS under basal conditions and under conditions of physiological stress remained largely unknown.

In the current study we performed a detailed behavioural characterisation of a mouse line with residual Neat1 expression, which revealed a distinct phenotype whose core feature is the exaggerated response to physiological stress. Under stressful conditions, these mice adopt an active coping strategy ("fight-or-flight" response), instead of passive coping strategy or freezing typical for wild-type mice. In addition, Neat $1^{-1-}$ mice were found to have deficiencies in social interactionencounter with unfamiliar animal or its odour, i.e., acute social stress. Furthermore, these mice also present with abnormal rhythmic fluctuations of daily activity, which is in line with the recently reported role for Neat1 in the control of circadian genes ${ }^{65}$. Behavioural features detected in these mice, i.e., hyperactivity, reckless behaviours, disinhibition, impulsivity, are commonly found in psychiatric disorders, including dementia, bipolar disorder, schizophrenia, and attention deficit hyperactivity disorder $(\mathrm{ADHD})^{66-68}$. Consistently, altered NEAT1 expression is a common theme in multiple neurological conditions including dementia ${ }^{69}$, Alzheimer's disease ${ }^{70}$, Huntington's disease $^{71}$, schizophrenia ${ }^{72,73}$, and epilepsy ${ }^{13,62}$. Therefore, abnormal NEAT1 expression and/or regulation might underlie at least some of the symptoms and phenotypes in common neurological diseases.

We cannot completely exclude possible influence of decreased Neat1 expression in other organs and tissues, e.g., thyroid and gonads, on the behaviour phenotype observed in Neat $1^{-/-}$mice. However, we believe that this phenotype is of primarily neurological origin for three main reasons: i) good general health of $\mathrm{Neat1}^{-1-}$ mice, even during aging; ii) absence of differences between males and females in test performance suggestive of low/ absent hormonal dependence; iii) high specificity of stimulation that triggers abnormal behaviour.

Our study also reports detailed analysis of Neat1 expression and distribution in the mammalian CNS regions using in situ hybridisation. We found that in the murine CNS (adult brain and spinal cord), Neat1 is expressed in multiple cell types with higher levels in glial cells. An earlier transcriptomic analysis of purified cell types also reported higher Neat1 levels in glial cellsastrocytes and microglial cells ${ }^{74}$. In another study, Neat1 was also reported to be highly expressed in oligodendrocytes $^{72}$. It should be noted that although chromogenic detection-based RNAscope ${ }^{\circledR}$ ISH does not allow colocalisation analysis, it is an extremely sensitive method permitting detection of individual clusters of molecules (e.g. individual Neat1 dots in neurons) in histological 
samples. The latter was not possible using fluorescent Neat1 ISH due to high level of tissue autofluorescence (unpublished observations).

The longer isoform of Neat1, Neat1_2, is the main structural component of paraspeckles ${ }^{3}$. However, Neat1_2 expression is restricted to certain organs/tissues, such as the gut, and was not detected in the $\mathrm{CNS}^{14}$. Using RNASeq, we also show that Neat1_2 expression is barely detectable in the brain under basal conditions. However, a previous study demonstrated accumulation of Neat1_2 in the murine hippocampus after seizures with a peak at the $8 \mathrm{~h}$ time-point and still detectable after $24 \mathrm{~h}^{45}$, suggesting that the isoform switch can take place in neurons under certain (stressful) conditions. Our data from human cultured neurons also indicated that Neat1_2 expression is a late event during stress/recovery from stress. This result is consistent with the in vivo data ${ }^{45}$ and indicates that neurons, which do not express NEAT1_2 under basal conditions, require some time to initiate its expression (as compared to cells already expressing this isoform, e.g., stable cell lines ${ }^{5}$ ). However, in all our behaviour paradigms, the testing schedule had a very short timeline and was therefore unlikely to be affected by the lack of acute Neat1_2 synthesis in Neat $1^{-/}$mice. Thus we believe that the behavioural phenotype of Neat1 ${ }^{-/}$mice can be ascribed, with confidence, to the changes caused by the loss of the expression of the constitutive neuronal Neat1 isoform, Neat1_1.

Although NEAT1 has been heavily implicated in the function of the immune system and in inflammatory response $\mathrm{e}^{7,41,42}$, we show that its loss in mice does not result in a neuroinflammatory phenotype. This result is consistent with the role for NEAT1 as a stress-responsive transcript which would mediate/facilitate a response to an external trigger (pathogen) but likely does not have housekeeping immune functions.

It has long been known that deficits in the inhibitory GABAergic neurotransmission lead to behavioural changes in mice characterised by hyperactivity, panic attacks, impulsivity and generalisation of fear. Therefore, it was possible that the hyperactive phenotype detected in Neat $1^{-/-}$mice is caused by attenuated GABA system function. However, in RNA-Seq analysis, we found that only one member of GABA type A receptor complex Gabrr2 is downregulated and another one, Gabrd, is differently spliced in the cortex of $\mathrm{Neat}^{-1-}$ mice. Immunohistochemistry and western blot analysis also did not reveal any detectable changes in the populations of GABAergic neurons throughout the brain of Neat $1^{-/-}$ mice. While it cannot be excluded that subtle changes in the GABAergic neurotransmission are still present in Neat $1^{-1-}$ mice, we concluded that it is not the main driver of the observed behaviour changes. Another possibility was that neurons derived from Neat $1^{-/-}$mice are intrinsically hyperexcitable, which was indeed the case and in line with previous data obtained in human neurons with acutely downregulated NEAT $1^{13}$. Augmented neuronal activity upon depletion of NEAT1 was previously determined indirectly, using calcium measurements. In our study, we used the gold standard method for this type of studies-whole-cell patch clamp recording in cultured neurons-which allowed to unambiguously establish the enhanced activity of $\mathrm{Neat}^{-/-}$neurons. Comparison of the electric properties showed that increased excitability of $\mathrm{Neat1}^{-/-}$neurons was likely due to the upregulated voltage-gated $\mathrm{Na}^{+}$influx, which determines the kinetics of the action potential and frequency of firing. Two-fold enlargement of voltage range availability for $\mathrm{Na}^{+}$current in $\mathrm{Neat1}^{-1-}$ neurons presumably allows greater number of these neurons to generate spontaneous and induced action potentials, whereas a trend for enhanced $\mathrm{K}^{+}$conductance $N e a t 1^{-1-}$ neurons could be crucial for faster repolarisation and therefore shortening of the absolute refractory period. Finally, we were able to demonstrate the relevant consequence of enhanced neuronal excitabilityincreased stress-induced cFos expression-in live mice. Our in vivo cFos data are consistent with a recent finding that Neat1 inhibits the expression of $c F o s$ in cultured neurons ${ }^{12}$. Notably, basal cFos expression was not changed in the brain of $\mathrm{Neat1}^{-/-}$mice in our RNA-Seq analysis, therefore cFos regulation by Neat1 mainly occurs upon stimulation, at least in vivo.

In an attempt to delineate molecular (gene expression) changes which underlie the hyperexcitability phenotype in Neat $1^{-/}$neurons, we analysed differential gene expression and changes in the alternative splicing in the cortex of $\mathrm{Neat}^{-1-}$ mice. DEG analysis revealed only a small number of significantly changed genes, and prominent changes were found in the expression of genes located in the vicinity of Neat1 such as Frmnd8, Cd6 and Malat1, similar to a previous study ${ }^{72}$. This result is in agreement with the emerging role for lncRNAs as important regulators of gene expression in cis. Indeed, a large proportion of lncRNAs with a known function are now classed as cis-acting lncRNAs, including NEAT1 and MALAT $1^{75}$. Importantly, the distances covered by such RNAs can vary significantly $^{75}$, consistent with the fact that some genes affected by Neat1 loss of expression, albeit on the same chromosome as Neat1 locus, are not located in the vicinity of the locus. We cannot completely exclude the possibility that the targeting strategy used to disrupt the Neat 1 locus in this mouse model affected the genomic environment, however the cis-regulatory function of Neat1 probably still plays a significant role in the regulation of DEGs identified in our analysis. Unexpectedly, we found that the majority of DEGs $(\sim 90 \%)$ in Neat $1^{-1-}$ 
mice were downregulated, and this finding was further reproduced in an independent mouse cohort. Both NEAT1 and MALAT1 have been reported to be localised to numerous genomic sites and bind active genes ${ }^{76}$. Thus Neat1 likely contributes to positive regulation of gene expression, explaining why its loss results in attenuated transcription of multiple targets. It is conceivable that moderate Malat1 downregulation found in Neat $1^{-/-}$mice synergises with Neat1 loss to mediate this phenotype. In future studies, it will be important to characterise possible neurological changes in Malat1 knockout mice at the behavioural and molecular levels.

Alternative splicing occurs at high frequency in the CNS and is crucial for normal functioning of the brain ${ }^{77}$. Indeed, global changes in alternative splicing pattern have been identified in the brain of patients with psychiatric diseases such as schizophrenia ${ }^{78}$. In our RNA-Seq dataset, we also detected a number of genes differentially spliced in the cortex of $N e a t 1^{-/-}$mice. Functionally, these genes can be subdivided into groups related to synaptic functions, $m R N A$ processing and cancer. Even though we did not detect major synaptic abnormalities in Neat ${ }^{-/-}$mice, it is plausible that these alternative splicing changes translate into functional changes in the synapse and contribute to the observed behavioural phenotype. It is important to note that, although alternative splicing changes at the level of individual genes were subtle in these mice, small changes in multiple genes belonging to the same pathways likely resulted in a measurable phenotype. The role of lncRNAs in neural plasticity is emerging, the latter being crucial for stress-coping mechanisms and behavioural adaptation. For example, in response to repeated social defeat stress, hundreds of lncRNAs change their expression in the prefrontal cortex in mice, and these IncRNAs are involved in the regulation of synaptic function $^{79}$. NEAT1 interacts with multiple RNA-binding proteins $^{80}$ many of which are autoregulated, including via alternative splicing ${ }^{81}$. Therefore, Neat1 loss can also lead to dysregulation of large RNA-binding protein networks. Finally, the pathophysiological connection between Neat1 isoform expression and tumorigenesis is well-established, and our data suggest that at least some of the cancer-related network changes caused by altered Neat1 expression may be attributed to abnormal alternative splicing regulation ${ }^{82}$. Further studies are needed to determine whether the effects of Neat1 loss on gene expression and alternative splicing are specific for the CNS tissue.

Unlike CNS-specific lncRNAs, Neat1 is expressed in a variety of tissues. Presumably, such lncRNAs play a universal regulatory role, while the specificity arises from the repertoire of cell type-depended targets and environmental cues. It can be concluded that although Neat1 is not essential for basic brain functions, its availability becomes important in the presence of biologically significant stress stimuli. Overall, Neat1 plays a fine-tuning regulatory role in the nervous system and participates in the processes responsible for environmentally appropriate behaviour.

\section{Acknowledgements}

The study was supported by fellowships from Medical Research Foundation and Motor Neurone Disease Association (Shelkovnikova/Oct17/968-799) to TAS and by a grant from RFBR (20-34-70059) to MSK. HA is a recipient of Cardiff University/China Scholarship Council PhD studentship. We thank Polina Yarova for the help with calcium imaging. DNA sequencing was undertaken at the Cardiff University School of Biosciences Genomics Research Hub.

\section{Author details}

${ }^{1}$ School of Biosciences, Cardiff University, Cardiff CF10 3AX, UK. ${ }^{2}$ Institute of Physiologically Active Compounds of Russian Academy of Sciences, Chernogolovka 142432, Russian Federation. ${ }^{3}$ Medicines Discovery Institute, Cardiff University, Cardiff CF10 3AT, UK. ${ }^{4}$ School of Dental Sciences, Newcastle University, Newcastle upon Tyne NE2 4BW, UK. ${ }^{5}$ Sheffield Institute for Translational Neuroscience, University of Sheffield, Sheffield S10 2HQ, UK. ${ }^{6}$ Department of Biosciences, Durham University, Durham DH1 3LE, UK. ${ }^{7}$ Faculty of Pharmaceutical Sciences, Hokkaido University, Sapporo 060-0812, Japan

\section{Conflict of interest}

The authors declare that they have no conflict of interest.

\section{Publisher's note}

Springer Nature remains neutral with regard to jurisdictional claims in published maps and institutional affiliations.

Supplementary Information accompanies this paper at (https://doi.org/ 10.1038/s41398-020-0854-2).

Received: 16 December 2019 Revised: 10 May 2020 Accepted: 18 May 2020 Published online: 28 May 2020

\section{References}

1. Fox, A. H., Nakagawa, S., Hirose, T. \& Bond, C. S. Paraspeckles: where long noncoding RNA meets phase separation. Trends Biochem. Sci. 43, 124-135 (2018).

2. Fox, A. H. \& Lamond, A. I. Paraspeckles. Cold Spring Harb. Perspect. Biol. 2, a000687 (2010).

3. Sunwoo, H. et al. MEN epsilon/beta nuclear-retained non-coding RNAs are upregulated upon muscle differentiation and are essential components of paraspeckles. Genome Res. 19, 347-359 (2009).

4. Li, R., Harvey, A. R., Hodgetts, S. I. \& Fox, A. H. Functional dissection of NEAT1 using genome editing reveals substantial localization of the NEAT1_1 isoform outside paraspeckles. RNA 23, 872-881 (2017).

5. An, H., Tan, J. T. \& Shelkovnikova, T. A. Stress granules regulate stress-induced paraspeckle assembly. J. Cell Biol. 218, 4127-4140 (2019).

6. Choudhry, H. et al. Tumor hypoxia induces nuclear paraspeckle formation through HIF-2alpha dependent transcriptional activation of NEAT1 leading to cancer cell survival. Oncogene 34, 4546 (2015).

7. Imamura, $K$. et al. Long noncoding RNA NEAT1-dependent SFPQ relocation from promoter region to paraspeckle mediates IL8 expression upon immune stimuli. Mol. Cell 53, 393-406 (2014).

8. Lellahi, S. M. et al. The long noncoding RNA NEAT1 and nuclear paraspeckles are up-regulated by the transcription factor HSF1 in the heat shock response. J. Biol. Chem. 293, 18965-18976 (2018).

9. Wang, Y. et al. Genome-wide screening of NEAT1 regulators reveals crossregulation between paraspeckles and mitochondria. Nat. Cell Biol. 20, 1145-1158 (2018).

10. Hirose, T. et al. NEAT1 long noncoding RNA regulates transcription via protein sequestration within subnuclear bodies. Mol. Biol. Cell 25, 169-183 (2014).

11. Chakravarty, D. et al. The oestrogen receptor alpha-regulated IncRNA NEAT1 is a critical modulator of prostate cancer. Nat. Commun. 5, 5383 (2014).

12. Butler, A. A., Johnston D. R., Kaur, S. \& Lubin, F. D. Long noncoding RNA NEAT1 mediates neuronal histone methylation and age-related memory impairment. Sci. Signal. 12, eaaw9277 (2019). 
13. Barry, G. et al. The long non-coding RNA NEAT1 is responsive to neuronal activity and is associated with hyperexcitability states. Sci. Rep. 7, 40127 (2017).

14. Nakagawa, S., Naganuma, T., Shioi, G. \& Hirose, T. Paraspeckles are subpopulation-specific nuclear bodies that are not essential in mice. J. Cell Biol. 193, 31-39 (2011)

15. An, H., Williams, N. G. \& Shelkovnikova, T. A. NEAT1 and paraspeckles in neurodegenerative diseases: a missing Inc found? Noncoding RNA Res. 3, 243-252 (2018)

16. Nakagawa, S. et al. The IncRNA Neat1 is required for corpus luteum formation and the establishment of pregnancy in a subpopulation of mice. Development 141, 4618-4627 (2014).

17. Komada, M., Takao, K. \& Miyakawa, T. Elevated plus maze for mice. J. Vis. Exp. 22, e1088.

18. Walf, A. A. \& Frye, C. A. The use of the elevated plus maze as an assay of anxiety-related behavior in rodents. Nat. Protoc. 2, 322-328 (2007).

19. Hughes, R. N. The value of spontaneous alternation behavior (SAB) as a test of retention in pharmacological investigations of memory. Neurosci. Biobehav. Rev. 28, 497-505 (2004).

20. Dellu, F., Contarino, A., Simon, H., Koob, G. F. \& Gold, L. H. Genetic differences in response to novelty and spatial memory using a two-trial recognition task in mice. Neurobiol. Learn Mem. 73, 31-48 (2000).

21. Winslow, J. T. Mouse social recognition and preference. Curr. Protoc. Neurosci. 22, 8-16 (2003).

22. Koolhaas, J. M. et al. The resident-intruder paradigm: a standardized test for aggression, violence and social stress. J. Vis. Exp. 77, e4367 (2013).

23. Dobin, A. et al. STAR: ultrafast universal RNA-seq aligner. Bioinformatics 29 15-21 (2013).

24. Love, M. I., Huber, W. \& Anders, S. Moderated estimation of fold change and dispersion for RNA-seq data with DESeq2. Genome Biol. 15, 550 (2014).

25. Thorvaldsdottir, H., Robinson, J. T. \& Mesirov, J. P. Integrative genomics viewer (IGV): high-performance genomics data visualization and exploration. Brief. Bioinform. 14, 178-192 (2013).

26. Li, Y. I. et al. Annotation-free quantification of RNA splicing using LeafCutter. Nat. Genet. 50, 151-158 (2018).

27. Chen, E. Y. et al. Enrichr: interactive and collaborative HTML5 gene list enrichment analysis tool. BMC Bioinform. 14, 128 (2013).

28. Kuleshov, M. V. et al. Enrichr: a comprehensive gene set enrichment analysis web server 2016 update. Nucleic Acids Res. 44, W90-W97 (2016).

29. Peters, O. M. et al. Selective pattern of motor system damage in gammasynuclein transgenic mice mirrors the respective pathology in amyotrophic lateral sclerosis. Neurobiol. Dis. 48, 124-131 (2012).

30. Shelkovnikova, T. A. et al. Chronically stressed or stress-preconditioned neurons fail to maintain stress granule assembly. Cell Death Dis. 8, e2788 (2017).

31. Kukharsky, M. S. et al. Calcium-responsive transactivator (CREST) protein shares a set of structural and functional traits with other proteins associated with amyotrophic lateral sclerosis. Mol. Neurodegener. 10, 20 (2015).

32. Hamill, O. P., Marty, A., Neher, E., Sakmann, B. \& Sigworth, F. J. Improved patchclamp techniques for high-resolution current recording from cells and cell-free membrane patches. Pflug. Arch. 391, 85-100 (1981).

33. Telezhkin, V. et al. Forced cell cycle exit and modulation of GABAA, CREB, and GSK3beta signaling promote functional maturation of induced pluripotent stem cell-derived neurons. Am. J. Physiol. Cell Physiol. 310, C520-C541 (2016).

34. Garcia-Cabezas, M. A., John, Y. J., Barbas, H. \& Zikopoulos, B. Distinction of neurons, glia and endothelial cells in the cerebral cortex: an algorithm based on cytological features. Front. Neuroanat. 10, 107 (2016).

35. Naganuma, T. et al. Alternative $3^{\prime}$-end processing of long noncoding RNA initiates construction of nuclear paraspeckles. EMBO J. 31, 4020-4034 (2012).

36. Ferguson, S. A. \& Gray, E. P. Aging effects on elevated plus maze behavior in spontaneously hypertensive, Wistar-Kyoto and Sprague-Dawley male and female rats. Physiol. Behav. 85, 621-628 (2005).

37. Pawlak, C. R., Karrenbauer, B. D., Schneider, P. \& Ho, Y. J. The elevated plusmaze test: differential psychopharmacology of anxiety-related behavior. Emot. Rev. 4, 98-115 (2012).

38. Shoji, H., Takao, K., Hattori, S. \& Miyakawa, T. Age-related changes in behavior in C57BL/6J mice from young adulthood to middle age. Mol. Brain 9, 11 (2016).

39. Holmes, A., Parmigiani, S., Ferrari, P. F., Palanza, P. \& Rodgers, R. J. Behavioral profile of wild mice in the elevated plus-maze test for anxiety. Physiol. Behav. 71, 509-516 (2000).

40. Rodgers, R. J. \& Dalvi, A. Anxiety, defence and the elevated plus-maze. Neurosci. Biobehav. Rev. 21, 801-810 (1997).
41. Gast, M. et al. Long noncoding RNA NEAT1 modulates immune cell functions and is suppressed in early onset myocardial infarction patients. Cardiovasc. Res. 115, 1886-1906 (2019).

42. Morchikh, M. et al. HEXIM1 and NEAT1 long non-coding RNA form a multisubunit complex that regulates DNA-mediated innate immune response. Mol. Cell 67, 387-399 e385 (2017).

43. Zhang, J. et al. c-fos regulates neuronal excitability and survival. Nat. Genet. 30, 416-420 (2002).

44. Melia, K. R., Ryabinin, A. E., Schroeder, R., Bloom, F. E. \& Wilson, M. C. Induction and habituation of immediate early gene expression in rat brain by acute and repeated restraint stress. J. Neurosci. 14, 5929-5938 (1994).

45. Bluthgen, N., van Bentum, M., Merz, B., Kuhl, D. \& Hermey, G. Profiling the MAPK/ERK dependent and independent activity regulated transcriptional programs in the murine hippocampus in vivo. Sci. Rep. 7, 45101 (2017).

46. Zhang, B. et al. The IncRNA Malat1 is dispensable for mouse development but its transcription plays a cis-regulatory role in the adult. Cell Rep. 2, 111-123 (2012).

47. Nakagawa, S. et al. Malat1 is not an essential component of nuclear speckles in mice. RNA 18, 1487-1499 (2012).

48. Johnson, J. O. et al. Exome sequencing reveals riboflavin transporter mutations as a cause of motor neuron disease. Brain 135, 2875-2882 (2012).

49. Hirano, A., Braas, D., Fu, Y. H. \& Ptacek, L. J. FAD regulates cryptochrome protein stability and circadian clock in mice. Cell Rep. 19, 255-266 (2017).

50. Uezato, A. et al. Genetic and molecular risk factors within the newly identified primate-specific exon of the SAP97/DLG1 gene in the 3 q29 schizophreniaassociated locus. Am. J. Med. Genet. B 174, 798-807 (2017).

51. Lee, $Y$. et al. Microduplications disrupting the MYT1L gene (2p25.3) are associated with schizophrenia. Psychiatr. Genet. 22, 206-209 (2012).

52. Meyer, K. J., Axelsen, M. S., Sheffield, V. C., Patil, S. R. \& Wassink, T. H. Germline mosaic transmission of a novel duplication of PXDN and MYT1L to two male half-siblings with autism. Psychiatr. Genet. 22, 137-140 (2012).

53. Wang, $T$. et al. De novo genic mutations among a Chinese autism spectrum disorder cohort. Nat. Commun. 7, 13316 (2016).

54. Hohoff, C. et al. Association analysis of Rgs7 variants with panic disorder. J. Neural Transm. 116, 1523-1528 (2009).

55. Cooper-Knock, J. et al. Gene expression profiling in human neurodegenerative disease. Nat. Rev. Neurol. 8, 518-530 (2012).

56. Limpitikul, W. B., Dick, I. E., Ben-Johny, M. \& Yue, D. T. An autism-associated mutation in CaV1.3 channels has opposing effects on voltage- and $\mathrm{Ca}(2$ +)-dependent regulation. Sci. Rep. 6, 27235 (2016).

57. Grieco, G. S. et al. New CACNA1A deletions are associated to migraine phenotypes. J. Headache Pain. 19, 75 (2018).

58. Jen, J. C. \& Wan, J. Episodic ataxias. Handb. Clin. Neurol. 155, 205-215 (2018)

59. Martin, S. et al. De novo variants in GRIA4 lead to intellectual disability with or without seizures and gait abnormalities. Am. J. Hum. Genet. 101, 1013-1020 (2017).

60. Makino, C. et al. Positive association of the AMPA receptor subunit GluR4 gene (GRIA4) haplotype with schizophrenia: linkage disequilibrium mapping using SNPs evenly distributed across the gene region. Am. J. Med. Genet. B 116B, 17-22 (2003).

61. MacDonald, M. L. et al. Altered glutamate protein co-expression network topology linked to spine loss in the auditory cortex of schizophrenia. Biol. Psychiatry 77, 959-968 (2015).

62. Lipovich, L. et al. Activity-dependent human brain coding/noncoding gene regulatory networks. Genetics 192, 1133-1148 (2012).

63. Briggs, J. A., Wolvetang, E. J., Mattick, J. S., Rinn, J. L. \& Barry, G. Mechanisms of long non-coding RNAs in mammalian nervous system development, plasticity, disease, and evolution. Neuron 88, 861-877 (2015).

64. Wang, A., Wang, J., Liu, Y. \& Zhou, Y. Mechanisms of long non-coding RNAs in the assembly and plasticity of neural circuitry. Front. Neural Circuits 11, 76 (2017).

65. Torres, M. et al. Circadian RNA expression elicited by 3'-UTR IRAlu-paraspeckle associated elements. Elife 5, e14837 (2016).

66. Yen, Y. C. et al. Co-segregation of hyperactivity, active coping styles, and cognitive dysfunction in mice selectively bred for low levels of anxiety. Front. Behav. Neurosci. 7, 103 (2013).

67. Russell, V. A. Overview of animal models of attention deficit hyperactivity disorder (ADHD). Curr. Protoc. Neurosci. 54, 9-35 (2011).

68. Beyer, D. K. E. \& Freund, N. Animal models for bipolar disorder: from bedside to the cage. Int. J. Bipolar Disord. 5, 35 (2017). 
69. Tollervey, J. R. et al. Characterizing the RNA targets and position-dependent splicing regulation by TDP-43. Nat. Neurosci. 14, 452-458 (2011).

70. Puthiyedth, N., Riveros, C., Berretta, R. \& Moscato, P. Identification of differentially expressed genes through integrated study of Alzheimer's disease Affected brain regions. PLOS ONE 11, e0152342 (2016).

71. Sunwoo, J. S. et al. Altered expression of the long noncoding RNA NEAT1 in Huntington's disease. Mol. Neurobiol. 54, 1577-1586 (2016).

72. Katsel, P. et al. The expression of long noncoding RNA NEAT1 is reduced in schizophrenia and modulates oligodendrocytes transcription. NPJ Schizophr. 5, 3 (2019).

73. Li, J. et al. Relationship between schizophrenia and changes in the expression of the long non-coding RNAs Meg3, Miat, Neat1 and Neat2. J. Psychiatr. Res. 106, 22-30 (2018).

74. Dong, $X$. et al. Comprehensive identification of long non-coding RNAs in purified cell types from the brain reveals functional LncRNA in OPC fate determination. PLoS Genet 11, e1005669 (2015).

75. Gil, N. \& Ulitsky, I. Regulation of gene expression by cis-acting long non-coding RNAs. Nat. Rev. Genet. 21, 102-117 (2020).
76. West, J. A. et al. The long noncoding RNAs NEAT1 and MALAT1 bind active chromatin sites. Mol. Cell 55, 791-802 (2014).

77. Yeo, G., Holste, D., Kreiman, G. \& Burge, C. B. Variation in alternative splicing across human tissues. Genome Biol. 5, R74 (2004).

78. Reble, E., Dineen, A. \& Barr, C. L. The contribution of alternative splicing to genetic risk for psychiatric disorders. Genes Brain Behav. 17, e12430 (2018)

79. Wang, $X$. et al. RNA-sequencing and bioinformatics analysis of long noncoding RNAs and mRNAs in the prefrontal cortex of mice following repeated social defeat stress. Biomed. Res. Int. 2019, 7505260 (2019).

80. Nakagawa, S., Yamazaki, T. \& Hirose, T. Molecular dissection of nuclear paraspeckles: towards understanding the emerging world of the RNP milieu. Open Biol. 8, 180150 (2018).

81. Jangi, M., Boutz, P. L., Paul, P. \& Sharp, P. A. Rbfox2 controls autoregulation in RNA-binding protein networks. Genes Dev. 28, 637-651 (2014).

82. Liu, S. \& Cheng, C. Alternative RNA splicin||lg and cancer. Wiley Interdiscip. Rev. RNA 4, 547-566 (2013). 